\title{
Post-critical $S s P m p$ and its applications to Virtual Deep Seismic Sounding (VDSS) - 2: 1-D imaging of the crust/mantle and joint constraints with receiver functions
}

\author{
Tianze Liu ${ }^{\oplus},{ }^{1}$ Simon L. Klemperer, ${ }^{1}$ Gabriel Ferragut ${ }^{1,2, *}$ and Chunquan $\mathrm{Yu}^{3, \dagger}$ \\ ${ }^{1}$ Department of Geophysics, Stanford University, Stanford, CA 94305, USA. E-mail: tianze@stanford.edu \\ ${ }^{2}$ Department of Geosciences, North Dakota State University, Fargo, ND 58105, USA \\ ${ }^{3}$ Seismological Laboratory, California Institute of Technology, Pasadena, CA 91125, USA
}

Accepted 2019 August 9. Received 2019 August 2; in original form 2019 February 19

\begin{abstract}
SUMMAR Y
Virtual Deep Seismic Sounding (VDSS) has emerged as a novel method to image the crustmantle boundary (CMB) and potentially other lithospheric boundaries. In Part 1, we showed that the arrival time and waveform of post-critical $S_{s} P m p$, the post-critical reflection phase at the CMB used in VDSS, is sensitive to several different attributes of the crust and upper mantle. Here, we synthesize our methodology of deriving Moho depth, average crustal $V_{p}$ and uppermost-mantle $V_{p}$ from single-station observations of post-critical $S s P m p$ under a 1-D assumption. We first verify our method with synthetics and then substantiate it with a case study using the Yellowknife and POLARIS arrays in the Slave Craton, Canada. We show good agreement of crustal and upper-mantle properties derived with VDSS with those given by previous active-source experiments and our own $P$ receiver functions (PRF) in our study area. Finally, we propose a PRF-VDSS joint analysis method to constrain average crustal $V_{p} / V_{s}$ ratio and composition. Our PRF-VDSS joint analysis shows that the southwest Slave Craton has an intermediate crustal composition, most consistent with a Mesoarchean age.
\end{abstract}

Key words: Body waves; Composition and structure of the continental crust; Cratons; Crustal imaging; North America; Wave scattering and diffraction.

\section{INTRODUCTION}

In recent years, Virtual Deep Seismic Sounding (VDSS) has been used to measure Moho depth in different areas (e.g. Tseng et al. 2009; Yu et al. 2012, 2016; Tian et al. 2015; Kang et al. 2016; Parker et al. 2016; Thompson et al. 2019). SsPmp, the seismic phase used in VDSS, originates when upcoming teleseismic $S$ waves convert to downgoing $P$ waves at the free surface (the virtual source), which then undergo post-critical reflection at or within the crustmantle boundary (CMB) and finally reach the receiver (Fig. 1). (Here we use CMB to denote the boundary or geological transition from crust to mantle, whether abrupt or a 'velocity-gradient zone' spanning many kilometres; and Moho to denote a seismological inference about this transition, typically given as a single depth.) The arrival time of $S s P m p$ relative to the direct $S$ arrival (or $S s$ ), hereafter $T_{\mathrm{VDSS}}$, is used to estimate the Moho depth at the reflection point, commonly assumed to be the midpoint between the virtual source

\footnotetext{
* Now at: Department of Earth Sciences, University of Oregon, Eugene, OR 97403, USA

${ }^{\dagger}$ Now at: Department of Earth and Space Sciences, Southern University of Science and Technology, Guangdong Sheng 518055, China
}

and receiver. Because VDSS uses teleseismic events, the incident $S$ wave is usually approximated as a plane wave with constant ray parameter. To enable post-critical reflection of SsPmp at the CMB, appropriate epicentral distances are $\sim 30-50^{\circ}$ (Yu et al. 2016), corresponding to ray parameter $p=\sim 0.1256-0.1409 \mathrm{~s} \mathrm{~km}^{-1}$ for a focal depth of $0 \mathrm{~km}\left(1 / p=\sim 7.10-7.96 \mathrm{~km} \mathrm{~s}^{-1}\right)$, and slightly smaller $p$ for larger focal depths (Fig. 2 in Liu et al., 2018, hereafter Part 1). Due to post-critical reflection at the CMB, SsPmp usually has amplitude comparable to $S s$ (Aki \& Richards 2002a, Fig. 1b), making it possible to construct a seismic profile with a single event recorded by an array. Because $S s P m p$ undergoes post-critical reflection at the CMB, its phase is changed relative to $S s$ (Fig. 1b; hereafter referred to as phase shift for simplicity and denoted $\left.\Phi_{\mathrm{VDSS}}\right)$, making it difficult to directly pick $T_{\text {VDSs }}$ from raw records. Previous studies (e.g. Tseng et al. 2009; Tian et al. 2015; Yu et al. 2016) used 1-D waveform fitting to measure $T_{\mathrm{VDSS}}$ and then found the trade-off relation between average crustal $V_{p}\left(V_{p}{ }^{\text {av }}\right)$ and thickness $H$ using the equation:

$T_{V D S S}=2 H \sqrt{\frac{1}{V_{p}^{a v 2}}-p^{2}}$,

where $p$ is the ray parameter of $S s$ and SsPmp. In Part 1, we used 1-D synthetic tests to show that whereas $T_{\mathrm{VDSS}}$ is primarily determined 
(a)

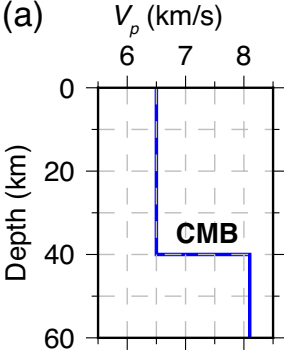

$V_{s}(\mathrm{~km} / \mathrm{s}) \quad \rho\left(\mathrm{g} / \mathrm{cm}^{3}\right)$

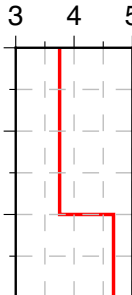

$\begin{array}{llll}5 & 2 & 3 & 4\end{array}$

(c)

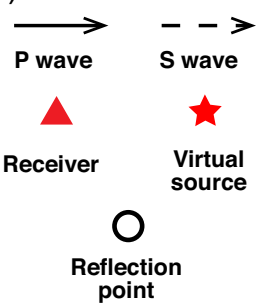

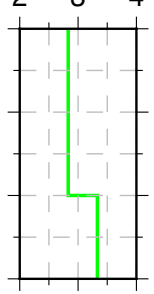

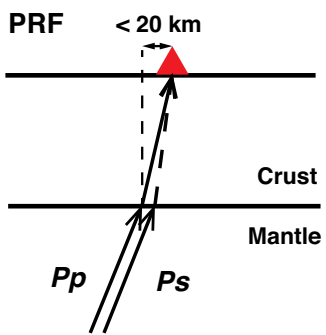

(b) Time (s)
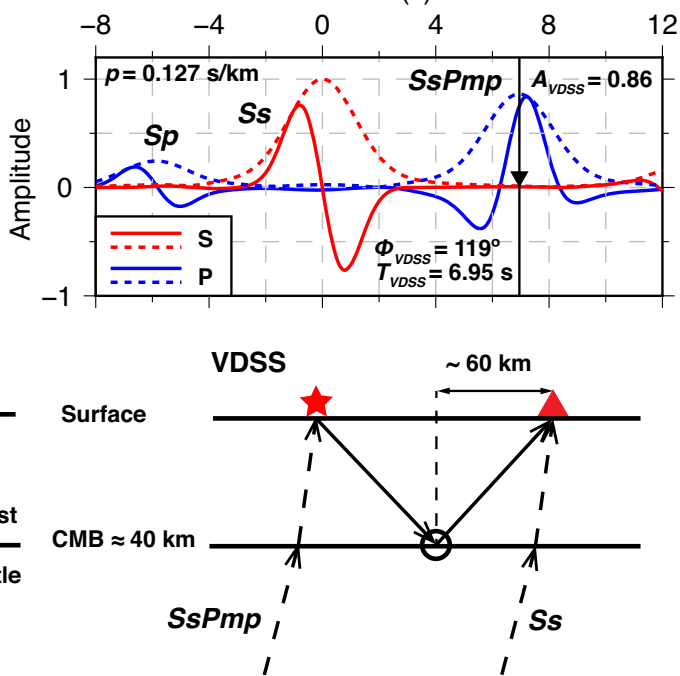

Figure 1. (a) 1-D $V_{p}, V_{s}$ and density model ('Model \#1'). (b) $P$ - and $S$-component synthetic seismograms ('observed waveforms') computed with Model \#1 for a ray parameter $p=0.127 \mathrm{~s} \mathrm{~km}^{-1}$ (epicentral distance of $\sim 48.5^{\circ}$ ). The dashed curves are corresponding envelope functions. Here and throughout this paper, $P$ components are blue and $S$ components red. Note the high amplitude and large phase shift of SsPmp relative to Ss. (c) Comparison between ray paths of $\mathrm{P}$ receiver functions (PRF) and Virtual Deep Seismic Sounding (VDSS).
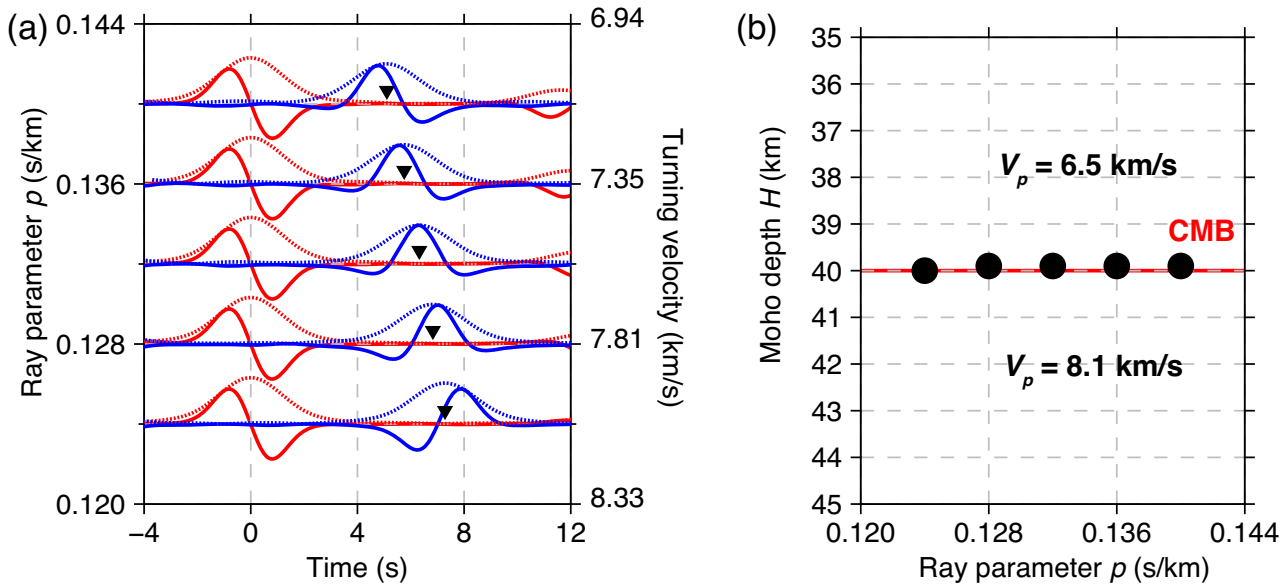

Figure 2. (a) Solid curves: $P$ - (blue) and $S$-component (red) synthetic waveforms ('observed waveforms') computed with Model \#1 for ray parameter $p=0.124-0.140 \mathrm{~s} \mathrm{~km}^{-1}$. Dotted curves: envelope functions. Black triangles: theoretical $T_{\mathrm{VDSS}}$ computed for Model \#1, always located at the peak of the $P$-component envelope functions despite the change in $\Phi_{\mathrm{VDSs}}$. (b) Black circles: Moho depths derived from $T_{\mathrm{VDSS}}$ estimated by picking peaks on $P$ envelope functions and converting to depth using the correct average crustal $V_{p}=6.5 \mathrm{~km} \mathrm{~s}^{-1}$. Red line: true CMB depth at $40 \mathrm{~km}$.

by CMB depth and $V_{p}^{\text {av }}, \Phi_{\mathrm{VDSs}}$ is sensitive to lower-crustal and uppermost-mantle $V_{p}\left(V_{p}^{\text {lc }}\right.$ and $\left.V_{p}{ }^{\text {um }}\right)$, which implies the possibility of constraining both $V_{p}^{\text {lc }}$ and $V_{p}$ um with observations of $\Phi_{\mathrm{VDSS}}$.

Here, we first show that we can estimate $T_{\mathrm{VDSS}}$ by picking peaks on SsPmp envelope functions and use it to derive Moho depth (Parker et al. 2016). We then present synthetic tests to demonstrate that $V_{p}$ um can be constrained without a priori knowledge of $V_{p}^{\text {lc }}$ using post-critical $S s P m p$ with turning velocity $1 / p$ close to $V_{p}$ um. In the presence of noise, we show that the estimation of $V_{p}$ um can be significantly improved by incorporating $\Phi_{\mathrm{VDSS}}$ measurements from multiple events. In addition, we propose a joint PRF-VDSS analysis scheme that simultaneously constrains $V_{p}{ }^{\text {av }}$ and average crustal $V_{p} / V_{s}$ ratio, thus crustal average composition $\left(V_{p} / V_{s}\right.$ ratio is also denoted $\kappa$, particularly when used in context of the $H-\kappa$ analysis of receiver functions: Zhu \& Kanamori 2000). Finally, we validate our proposed methods using the Yellowknife and POLARIS arrays in the SW Slave Craton, finding good agreement between the crustal and upper-mantle properties we derive with VDSS and those from previous studies using active-source experiments and our own $P$ receiver functions (PRF). We demonstrate with our PRF-VDSS joint analysis that the SW Slave Craton has an intermediate crustal composition.

\section{SYNTHETIC EXAMPLES}

\subsection{Measuring $T_{\text {VDSS }}$ from envelope functions}

When a signal undergoes a phase shift, its envelope function stays constant despite the changing waveform, (Aki \& Richards 2002a). Therefore, a simple way to account for the phase shift of SsPmp 
relative to $S s$ while measuring $T_{\mathrm{VDSS}}$ is to calculate the time between the peaks on $S s$ and $S s P m p$ envelope functions (Parker et al. 2016). Fig. 2(a) shows synthetic waveforms calculated using the reflectivity algorithm (Randall 1989) for the 1-D models in Fig. 1 (hereafter 'Model \#1') and $0.124 \leq p \leq 0.140 \mathrm{~s} \mathrm{~km}^{-1}$, after separation into pseudo- $P$ (motion associated with incoming $P$ waves) and pseudo- $S$ (motion associated with incoming $S$ waves) components (hereafter referred to as $P$ and $S$ for simplicity) with a particle-motion analysis algorithm (Yu et al. 2013) and converting to envelope functions. The shapes of $S s$ ( $S$ component) and $S s P m p$ ( $P$ component) envelope functions are essentially identical, as the arrivals are only distinguished by a phase shift ( $\left.\Phi_{\mathrm{VDSS}}\right)$, a negligible amplitude change due to post-critical reflection, and a time delay. We then measure $T_{\mathrm{VDSS}}$ from these $S s$ and $S s P m p$ envelope functions and convert them to Moho depths using eq. (1) and the true $V_{p}{ }^{\text {av }}$. The estimated Moho depths agree (within $0.1 \mathrm{~km}$ ) with the true CMB depth for all $p$ (Fig. 2b), indicating the robustness of this method. Given multiple SsPmp observations with a wide range of ray parameter $p$, one can simultaneously determine Moho depth and $V_{p}{ }^{\text {av }}$ (Kang et al. 2016), as shown below. Although $T_{\text {VDSS }}$ can be robustly estimated from SsPmp envelope functions, when calculating these envelope functions we effectively eliminate the information contained in $\Phi_{\text {VDSS }}$ (Fig. 2a), which is sensitive to the structure of the CMB (Part 1). Therefore, a more desirable way to analyse $S s P m p$ is to model $T_{\mathrm{VDSS}}$ and $\Phi_{\mathrm{VDSS}}$ simultaneously.

\subsection{Modeling $T_{\mathrm{VDSS}}$ and $\Phi_{\mathrm{VDSS}}$ simultaneously for a single event}

In order to model $T_{\mathrm{VDSS}}$ and $\Phi_{\mathrm{VDSS}}$ simultaneously, we first generate synthetic SsPmp waveforms for all combinations of $V_{p}$ in the lower crust $\left(V_{p}^{\text {lc }}\right)$ and upper mantle $\left(V_{p}\right.$ um $)$ using the reflection coefficient at the Moho (Aki \& Richards 2002b). We then use cross-correlation to align the synthetic $S s P m p$ waveforms with the 'observed' data (e.g. the waveforms in Fig. 1b) and compute the normalized misfit (hereafter referred to as misfit for simplicity) between the synthetic and 'observed' SsPmp. Before computing the misfit, we normalize the 'observed' and synthetic $S s P m p$ by the peak values of their envelope functions so that the effects of amplitude difference are eliminated. The synthetic $S s P m p$ waveform with the minimum misfit determines the best-fitting $V_{p}^{\text {lc }}$ and $V_{p}$ um, and the best-fitting $T_{\text {VDSS }}$ is found by picking the peak cross-correlation value between the best-fitting synthetic SsPmp and the 'observed' one. We note that previous 1-D modelling schemes have held $V_{p}^{\text {lc }}$ and/or $V_{p}$ um fixed (Tseng et al. 2009; Tian et al. 2015; Yu et al. 2016). To test our approach, we use the reflectivity method (Randall 1989) to compute 'observed' waveforms and try to recover $T_{\mathrm{VDSS}}, V_{p}{ }^{\text {lc }}$ and $V_{p}{ }^{\text {um }}$ from them using the method described above.

We first attempt to recover both $V_{p}^{\text {lc }}$ and $V_{p}$ um from the observed SsPmp waveforms. Our 'observed' waveforms are computed with Model \#1, a single layer crust with $V_{p}=6.5 \mathrm{~km} \mathrm{~s}^{-1}$ overlaying a half space with $V_{p}=8.1 \mathrm{~km} \mathrm{~s}^{-1}$ that represents the upper mantle (Fig. 1a). We use two different ray parameters $p=0.128$ and $0.136 \mathrm{~s} \mathrm{~km}^{-1}$ to study the potential effects of ray parameter. After applying our proposed method, the resulting misfits are plotted as functions of $V_{p}^{\text {lc }}$ and $V_{p}^{\text {um }}$ (Fig. 3). In the case with $p=0.128 \mathrm{~s} \mathrm{~km}^{-1}$ $\left(1 / p=7.81 \mathrm{~km} \mathrm{~s}^{-1}\right)$, we observe that the misfit depends strongly on $V_{p}$ um and weakly on $V_{p}^{\text {lc }}$, with the misfit contours having small neg-

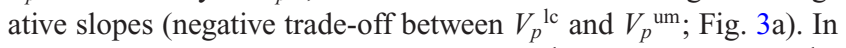
contrast, in the case with $p=0.136 \mathrm{~s} \mathrm{~km}^{-1}\left(1 / p=7.35 \mathrm{~km} \mathrm{~s}^{-1}\right)$, although the trade-off between $V_{p}^{\text {lc }}$ and $V_{p}$ um is still negative, the misfit depends weakly on both $V_{p}^{\text {lc }}$ and $V_{p}^{\text {um }}$ (Fig. 3b). [Note that because the observed and synthetic waveforms are not computed in the same way, minor differences exist between them that makes the minimum misfits in both cases non-zero (Fig. 3).] Because fitting SsPmp waveforms is equivalent to fitting $\Phi_{\mathrm{VDSS}}$, this behaviour of misfit can be understood by considering the dependence of $\Phi_{\mathrm{VDSS}}$ on $V_{p}^{\text {lc }}$ and $V_{p}$ um. When $1 / p$ is close to $V_{p}$ um, for example the case with $p=0.128 \mathrm{~s} \mathrm{~km}^{-1}\left(1 / p=7.81 \mathrm{~km} \mathrm{~s}^{-1}\right.$; Fig $\left.3 \mathrm{a}\right)$, $\Phi_{\mathrm{VDSs}}$ is primarily controlled by $V_{p}$ um with only weak dependence on $V_{p}^{\text {lc }}$ (Fig. 7a in Part 1). When $1 / p$ is close to neither $V_{p}$ um nor $V_{p}^{\text {lc }}$, for example the case with $p=0.136 \mathrm{~s} \mathrm{~km}^{-1}\left(1 / p=7.35 \mathrm{~km} \mathrm{~s}^{-1}\right.$; Fig. 3b), $\Phi_{\mathrm{VDSS}}$ depends weakly on both $V_{p}^{\text {um }}$ and $V_{p}^{\text {lc }}$ (Part 1 ). Ideally, when $1 / p$ is close to $V_{p}{ }^{\text {lc }}$, misfit will be primarily controlled by $V_{p}^{\text {lc }}$ due to the strong dependence of $\Phi_{\mathrm{VDSS}}$ on $V_{p}^{\text {lc }}$ (Fig. $7 \mathrm{~b}$ in Part 1). However, in this case the large $p$ may cause strong pre-critical reflections from intracrustal interfaces that may interfere with SsPmp and distort its waveform (e.g. Fig. 5 in Part 1). Therefore, a more practical way to utilize $\Phi_{\text {VDSS }}$ observations is to infer $V_{p}$ um from $\Phi_{\text {VDSS }}$ while assuming a known $V_{p}^{\text {lc }}$ in cases with $1 / p$ close to $V_{p}$ um, because in such cases an incorrect $V_{p}^{\text {lc }}$ would only have limited effect on estimated $V_{p}^{\text {um }}$ (Fig. 3a).

Fig. 4 shows examples of this strategy of estimating Moho depth and $V_{p}$ um from $S_{s} P m p$ waveforms while fixing $V_{p}{ }^{\text {lc }}$. We use the correct $V_{p}{ }^{\text {av }}=6.5 \mathrm{~km} \mathrm{~s}^{-1}$ in eq. (1) to convert the measured $T_{\mathrm{VDSS}}$ to Moho depth. For $p=0.128 \mathrm{~s} \mathrm{~km}^{-1}$, when the true $V_{p}^{\text {lc }}=6.5 \mathrm{~km} \mathrm{~s}^{-1}$ ( $V_{p}{ }^{\text {lc }}$ and $V_{p}{ }^{\text {av }}$ are the same here because the crust is homogeneous) is assumed, we are able to recover both the CMB depth and $V_{p}$ um precisely (Fig. 4a). Because we precisely recovered $V_{p}$ um and $V_{p}^{\text {lc }}$, the synthetic SsPmp matches the observation almost perfectly (Fig. 4b). For the same 'observed' data $\left(p=0.128 \mathrm{~s} \mathrm{~km}^{-1}\right)$, when we vary $V_{p}^{\text {lc }}$ from the true value to 6.2 and $6.8 \mathrm{~km} \mathrm{~s}^{-1}(\sim \pm 5$ per cent perturbation), only $\sim \pm 0.6$ per cent error is introduced in estimated $V_{p}{ }^{u m}\left(< \pm 0.05 \mathrm{~km} \mathrm{~s}^{-1}\right)$, and its effect on estimated Moho depth is negligible (Fig. 4c). The misfit curves for $V_{p}{ }^{\text {lc }}=6.2,6.5$ and $6.8 \mathrm{~km} \mathrm{~s}^{-1}$ have very similar minima, indicating that the synthetics fit the 'observed' data equally well for all three cases (Fig. 4d). Because $\Phi_{\text {VDSS }}$ is matched precisely in all three cases, the correct $T_{\mathrm{VDSS}}$ is found in each case and we are able to give the correct CMB depth despite errors in assumed $V_{p}^{\text {lc }}$ (Fig. 4c). However, when $p=0.136 \mathrm{~s} \mathrm{~km}^{-1}$, again varying $V_{p}^{\text {lc }}$ from 6.2 to $6.8 \mathrm{~km} \mathrm{~s}^{-1}$ causes $\sim \pm 1-2$ per cent error in the estimated $V_{p}$ um, significantly larger than for $p=0.128 \mathrm{~s} \mathrm{~km}^{-1}$, though the effect on estimated Moho depth remains negligible (Fig. 4e) due to precise matching of the SsPmp waveforms. We also observe that the troughs of the misfit curves are significantly wider for $p=0.136 \mathrm{~s} \mathrm{~km}^{-1}$ than for $p=0.128 \mathrm{~s} \mathrm{~km}^{-1}$, indicating poorer constraints on $V_{p}^{\text {um }}$ (Fig. 4f). These results show that, in a $1-D$ earth, matching $\Phi_{\text {VDSs }}$ alone is sufficient to estimate $T_{\mathrm{VDSS}}$ and Moho depth precisely, whereas to infer $V_{p}$ um from $\Phi_{\mathrm{VDSS}}$ requires a priori knowledge of $V_{p}{ }^{\text {lc }}$. If $V_{p}^{\text {lc }}$ is poorly constrained in the study region, it is better to use SsPmp waveforms with $1 / p$ close to $V_{p}$ um, so that an incorrectly assumed $V_{p}^{\text {lc }}$ causes less error in the estimated $V_{p}$ um. Even in cases with well-constrained $V_{p}^{\text {lc }}$, using $S s P m p$ waveforms with $1 / p$ close to $V_{p}$ um is still preferred because the narrower troughs of the misfit curves in such cases would result in better constrained $V_{p}$ um (smaller uncertainty; Supplementary Text 1).

Because in field data the $S$ wave trains are usually contaminated by $P$-wave coda, it is important to test our methods for cases with reasonable noise level. To simulate realistic noise, we generate Gaussian white noise, bandpass filter it between 0.05 and $0.5 \mathrm{~Hz}$ (a range typically used for filtering field data) and scale the noise 


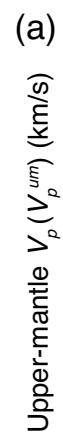

(a)
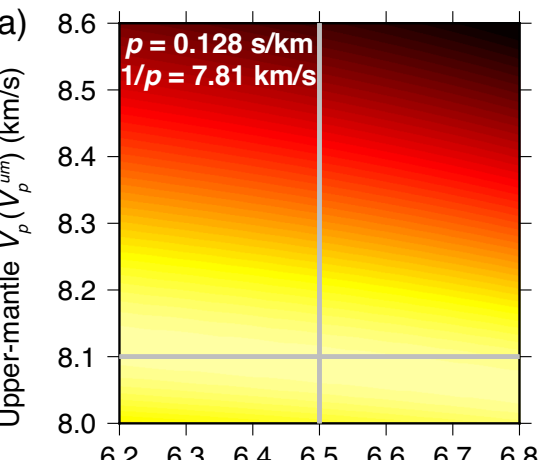

(b)

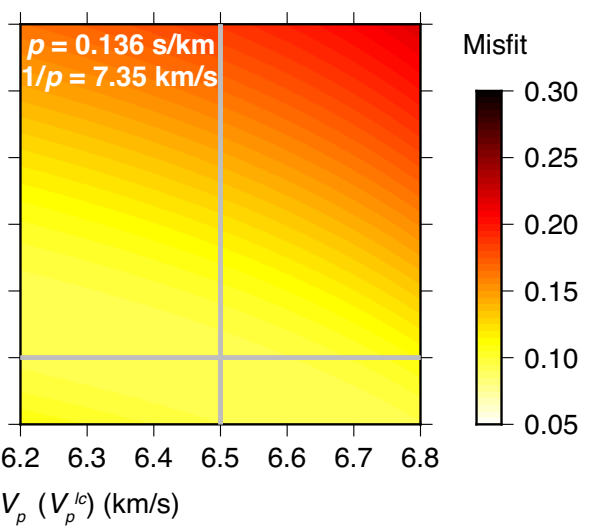

Figure 3. Normalized misfit between synthetic waveforms generated with a grid search in $V_{p}$ um $-V_{p}$ lc space and 'observed waveform' computed with Model $\# 1$ for (a) $p=0.128 \mathrm{~s} \mathrm{~km}^{-1}$ and (b) $p=0.136 \mathrm{~s} \mathrm{~km}^{-1}$. Best fit to the 'observed waveform' is always at the Model \#1 parameters (gray crosses), but $V_{p}^{\text {um is }}$

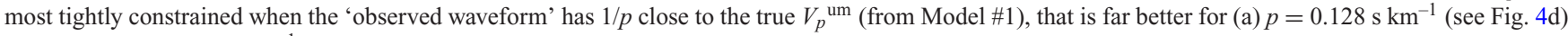
than for (b) $p=0.136 \mathrm{~s} \mathrm{~km}^{-1}$ (see Fig. 4f).

so that its standard deviation is 10 per cent of the peak amplitude on the radial-component data. We add random noise independently to the same radial- and vertical-component synthetic waveforms used in the noise-free case (Fig. 4) and again attempt to retrieve $V_{p}^{\text {um }}$ and Moho depth (Fig. S1). For $p=0.128 \mathrm{~s} \mathrm{~km}^{-1}$, the derived $V_{p}{ }^{\text {um }}$ and Moho depth are significantly biased due to the interference of noise with the SsPmp waveform (Figs S1a-d), whereas the two parameters are reasonably well-recovered for the case with $p=0.136 \mathrm{~s} \mathrm{~km}^{-1}$ (Figs S1e and $\mathrm{f}$ ). Since the added noise is random, the higher bias for the $p=0.128 \mathrm{~s} \mathrm{~km}^{-1}$ case is likely a coincidence (noise level happens to be higher in the time window of SsPmp). To demonstrate this, we generate 100 random simulations of noisy SsPmp both for $p=0.128 \mathrm{~s} \mathrm{~km}^{-1}$ and for $p=0.136 \mathrm{~s} \mathrm{~km}^{-1}$ and derive $V_{p}$ um from each simulation (Fig. S2). The mean estimated $V_{p}{ }^{\text {um }}$ from 100 simulations (equivalent to 100 earthquakes with the same $p$ recorded at a single station) are consistent with the true value both for $p=0.128 \mathrm{~s} \mathrm{~km}^{-1}$ and for $p=0.136 \mathrm{~s} \mathrm{~km}^{-1}$, even though individual simulations (single earthquakes) can produce significant errors (Fig. S2). Therefore, the larger error for $p=0.128 \mathrm{~s} \mathrm{~km}^{-1}$ in Fig. $\mathrm{S} 1$ is indeed coincidental. Estimated $V_{p}{ }^{\text {um }}$ has a larger standard deviation for $p=0.136 \mathrm{~s} \mathrm{~km}^{-1}$ than for $p=0.128 \mathrm{~s} \mathrm{~km}^{-1}$ (Fig. $\mathrm{S} 2$ ) due to higher sensitivity of $\Phi_{\mathrm{VDSS}}$ to $V_{p}$ um when $p=0.128 \mathrm{~s}$ $\mathrm{km}^{-1}$ (Figs 4 and $\mathrm{S} 1$ ). In order to avoid these errors due to random noise, an obvious strategy is to combine observations of post-critical SsPmp from multiple events in our estimation of Moho depth and $V_{p}^{\text {um }}$.

\subsection{Crustal and mantle properties from $T_{\mathrm{VDSS}}$ and $\Phi_{\mathrm{VDSS}}$ of multiple events}

To incorporate observations of multiple events, we first measure $T_{\mathrm{VDSS}}$ and $\Phi_{\mathrm{VDSS}}$ from individual events and then estimate crustal and mantle properties by modeling the observed $T_{\mathrm{VDSS}}$ and $\Phi_{\mathrm{VDSS}}$. In order to measure $T_{\text {VDSs }}$ and $\Phi_{\text {VDSS }}$ simultaneously, for each event we use a cosine-tapered 15-s window around the $S s$ waveform on the $\mathrm{S}$ component as the source wavelet of that event. We then apply phase shifts from $0-360^{\circ}$ to the source wavelet to create synthetic SsPmp waveforms with all possible phase shifts. We use crosscorrelation to find the best alignment between each synthetic $S s P m p$ wavelet and the observed SsPmp. We then normalize observed and synthetic SsPmp and compute the misfit between them. $\Phi_{\mathrm{VDSS}}$ is determined as the phase shift that minimizes the misfit between the synthetic and modeled $S s P m p$. We estimate the uncertainty of $T_{\mathrm{VDSS}}$ and $\Phi_{\mathrm{VDSS}}$ from the curvature of the misfit-phase-shift function around $\Phi_{\text {VDSS }}$ (Text S1 and Fig. S3). We then find $T_{\text {VDSS }}$ of each event by cross-correlation between the best-fit synthetic $S s P m p$ and the observed $S_{s} P m p$. After measuring $T_{\mathrm{VDSS}}$ and $\Phi_{\mathrm{VDSS}}$ for all events, we use the two parameters as functions of ray parameters to constrain crustal and mantle properties. Following Kang et al. (2016), we use $T_{\text {VDSS }}$ as a function of $p$ (the moveout of post-critical SsPmp) to constrain $V_{p}$ av and Moho depth $H$ with linear regression, which also gives uncertainties of estimated $V_{p}$ av and $H$ (Fig. 5c). In our linear regression procedure, we scale each data point with its uncertainty, so that data points with high uncertainty have smaller weight on the results. To constrain $V_{p}^{\text {um }}$, we compare observed $\Phi_{\mathrm{VDSS}}$ as a function of $p$ with theoretical $\Phi_{\mathrm{VDSs}}-p$ relations computed with a fixed $V_{p}^{\text {lc }}$ and a range of $V_{p}$ um (Fig. 5d).

To test our method, we generate synthetic SsPmp waveforms using Model \#1 and $p=0.124-0.134 \mathrm{~s} \mathrm{~km}^{-1}$, on which we can clearly observe a decrease in $T_{\text {VDSS }}$ and $\Phi_{\text {VDSS }}$ with increasing $p$ (Fig. 5a). We then measure $T_{\text {VDSS }}$ and $\Phi_{\text {VDSs }}$ from the observed $S s P m p$ of each event, an example of which is shown in Fig. 5(b). The observed $T_{\mathrm{VDSS}}-p$ relation gives a best-fitting $V_{p}{ }^{\text {av }}=6.5 \pm 0.1 \mathrm{~km} \mathrm{~s}^{-1}$ and $H=40 \pm 1 \mathrm{~km}$, in good agreement with the input model (Fig. 5c). To find the best-fitting $V_{p}$ um , we plot theoretical $\Phi_{\mathrm{VDSS}}-p$ relations with different $V_{p}{ }^{\text {um }}$ values while assuming $V_{p}{ }^{\text {lc }}=6.5 \mathrm{~km} \mathrm{~s}^{-1}$, the true $V_{p}{ }^{\text {lc }}$, and compare them with the observed values. The comparison shows that the observations favour $V_{p}{ }^{\text {um }}=8.1 \mathrm{~km} \mathrm{~s}^{-1}$, consistent with the input model (Fig. 5d). To test the effects of assumed $V_{p}^{\text {lc }}$ on the estimated $V_{p}$ um, we compare theoretical curves computed assuming $V_{p}^{\text {lc }}=6.2,6.5,6.8 \mathrm{~km} \mathrm{~s}^{-1}$ ( $\sim 5$ per cent perturbation; Fig. 6). For $V_{p}^{\mathrm{lc}}=6.2$ and $6.8 \mathrm{~km} \mathrm{~s}^{-1}$, the theoretical $\Phi_{\mathrm{VDSs}}-p$ curves overlap when $p$ is small and gradually diverge as $p$ increases (Fig. 6), but never to the extent that would significantly affect the estimated $V_{p}{ }^{\text {um }}$ (Fig. 6). The theoretical $\Phi_{\mathrm{VDSs}}-p$ relations are insensitive to assumed $V_{p}^{\text {lc }}$ because the reciprocal of ray parameter $(1 / p)$ considered here $\left(7.5-8.1 \mathrm{~km} \mathrm{~s}^{-1}\right)$ is significantly higher than the assumed $V_{p}^{\text {lc }}$, which makes $\Phi_{\text {VDSs }}$ insensitive to $V_{p}^{\text {lc }}$ (Fig. 7a in Part 1). In the case of $V_{p}{ }^{\text {lc }}=6.2 \mathrm{~km} \mathrm{~s}^{-1}\left(6.8 \mathrm{~km} \mathrm{~s}^{-1}\right)$, the theoretical $\Phi_{\mathrm{VDSs}}-$ $p$ curves are shifted slightly upward (downward) compared with $V_{p}{ }^{\text {lc }}=6.5 \mathrm{~km} \mathrm{~s}^{-1}$, because for a fixed $p$, decreasing (increasing) $V_{p}^{\text {lc }}$ while fixing $\Phi_{\mathrm{VDSS}}$ requires an increase (decrease) in $V_{p}$ um (negative trade-off between $V_{p}^{\text {lc }}$ and $V_{p}^{\text {um }}$; Fig. 7 in Part 1). 
Model \#1, $p=0.128 \mathrm{~s} / \mathrm{km}, \Delta=47.5^{\circ}$

(a)

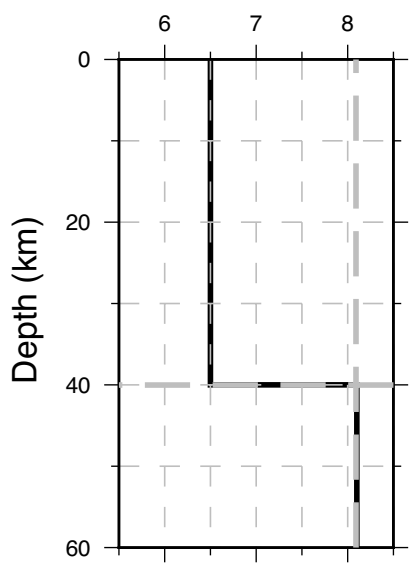

(b)

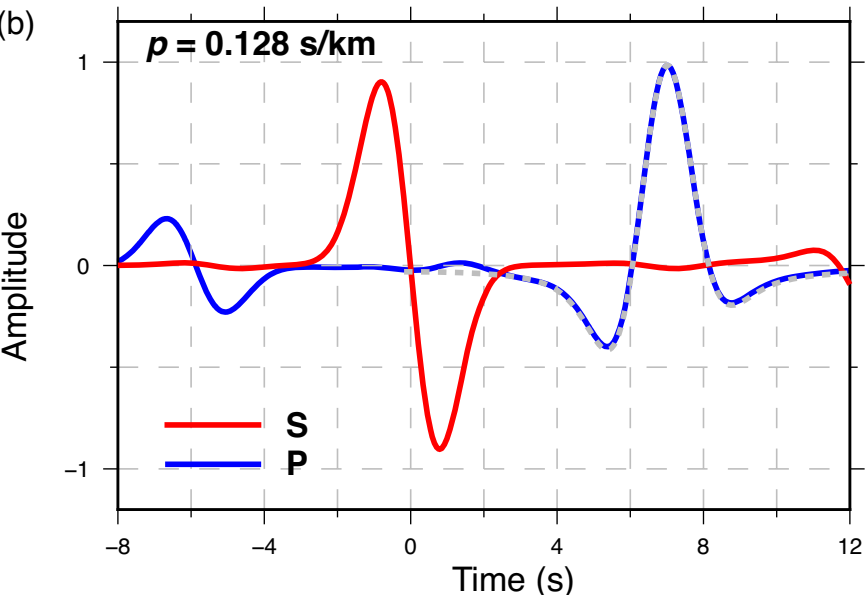

Model \#1, $p=0.128 \mathrm{~s} / \mathrm{km}, \Delta=47.5^{\circ}$

(c)

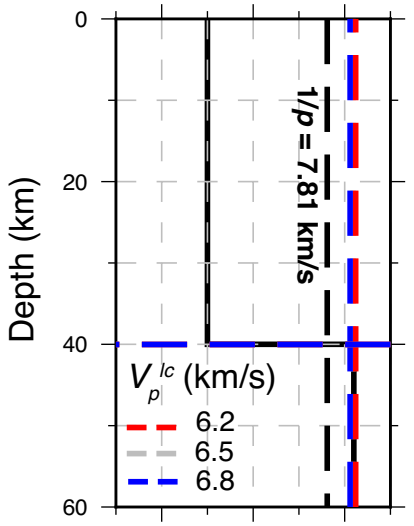

(d)

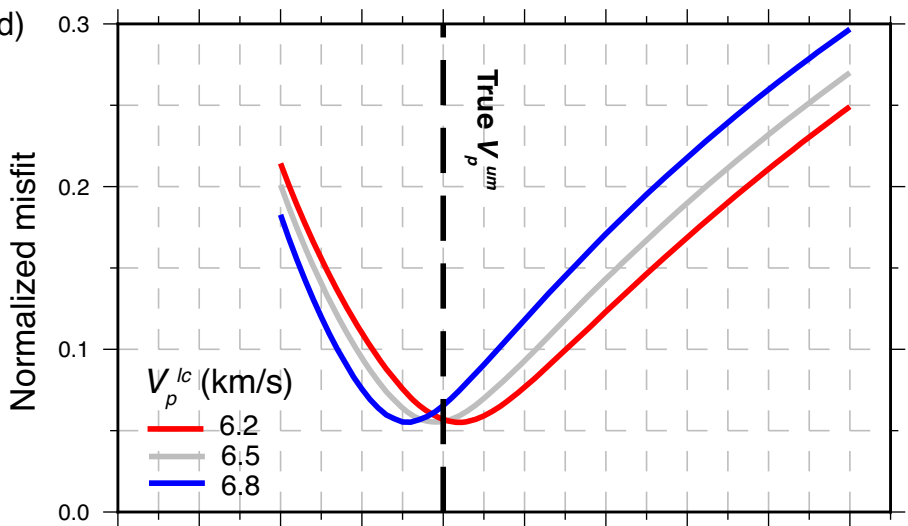

Model \#1, $p=0.136 \mathrm{~s} / \mathrm{km}, \Delta=38^{\circ}$

(e)

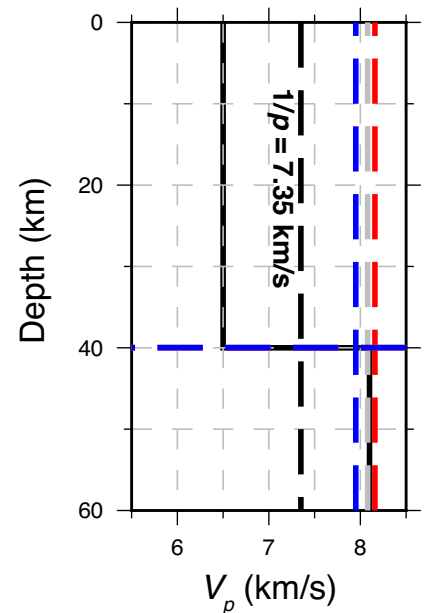

(f)

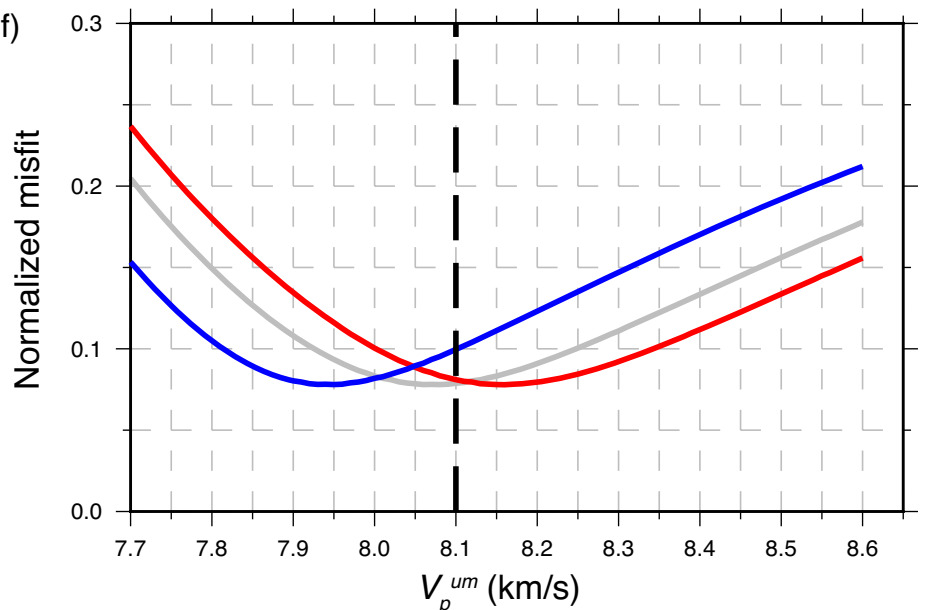

Figure 4. Estimating Moho depth and $V_{p}^{\text {um }}$ from waveforms computed for Model $\# 1$ with an assumed $V_{p}^{\text {lc }}$. (a) and (b) Moho depth and $V_{p}^{\text {um }}$ derived using the correct $V_{p}{ }^{\text {lc }}=6.5 \mathrm{~km} \mathrm{~s}^{-1}$. (a) Gray dashed lines: Moho depth and $V_{p}$ um that best fit $T_{\mathrm{VDSS}}$ and $\Phi_{\mathrm{VDSS}}$, very closely matching Model \#1 (black line). $V_{p}^{\text {av }}=6.5 \mathrm{~km} \mathrm{~s}^{-1}$ is used to convert $T_{\mathrm{VDSS}}$ to Moho depth. (b) Synthetic $P$ and $S$ waveforms computed for Model \#1 and $p=0.128 \mathrm{~s} \mathrm{~km}{ }^{-1}$ ('observations'). Grey dashed curve: $P$-component $S s P m p$ waveform that best fits $T_{\text {VDSS }}$ and $\Phi_{\text {VDSs. }}$ (c-f) A comparison of results derived with different assumed $V_{p}$ lc . (c) and

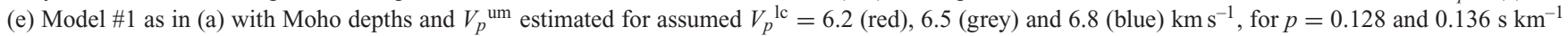
respectively. Black dashed lines mark the critical velocities $(1 / p)$ in the two cases. (d) and (f) Normalized misfits as functions of $V_{p}$ um for each case. Note the choice of $V_{p}^{\text {lc }}$ has little effect on estimated $V_{p}{ }^{\text {um }}$ when $p$ is small ( $1 / p$ is large and close to $V_{p}{ }^{\text {um }}$ ), but has a significant effect when $p$ is large ( $1 / p$ is small and far from $V_{p}^{\text {um }}$ ). 
(a)

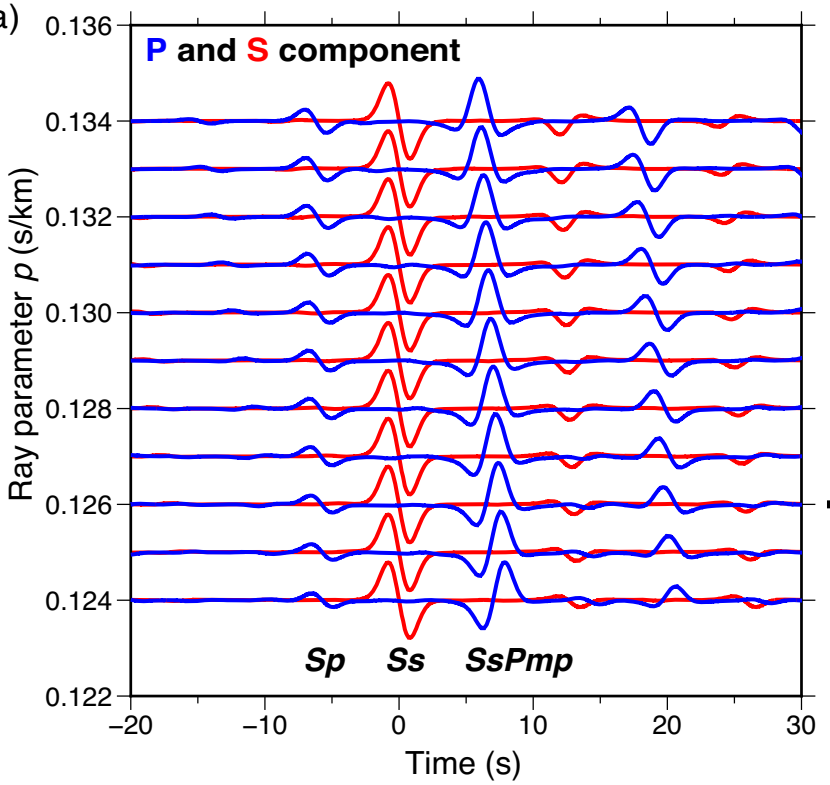

(c)

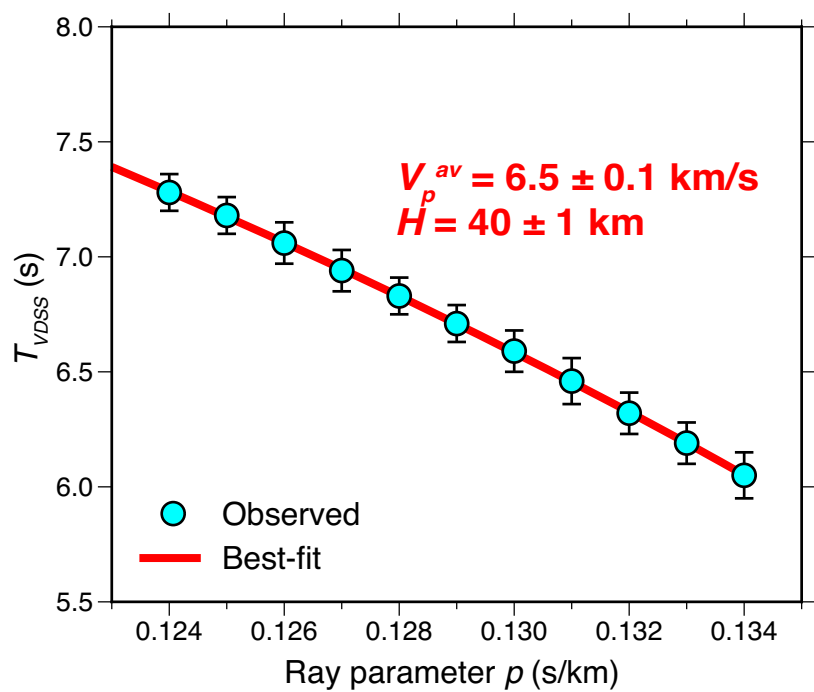

(b)

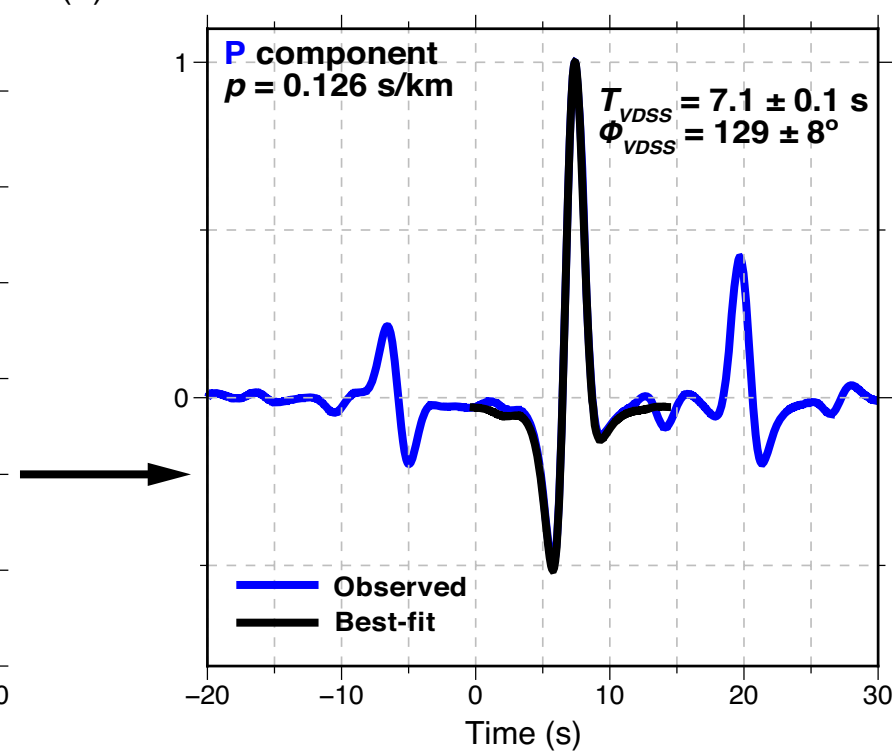

(d)

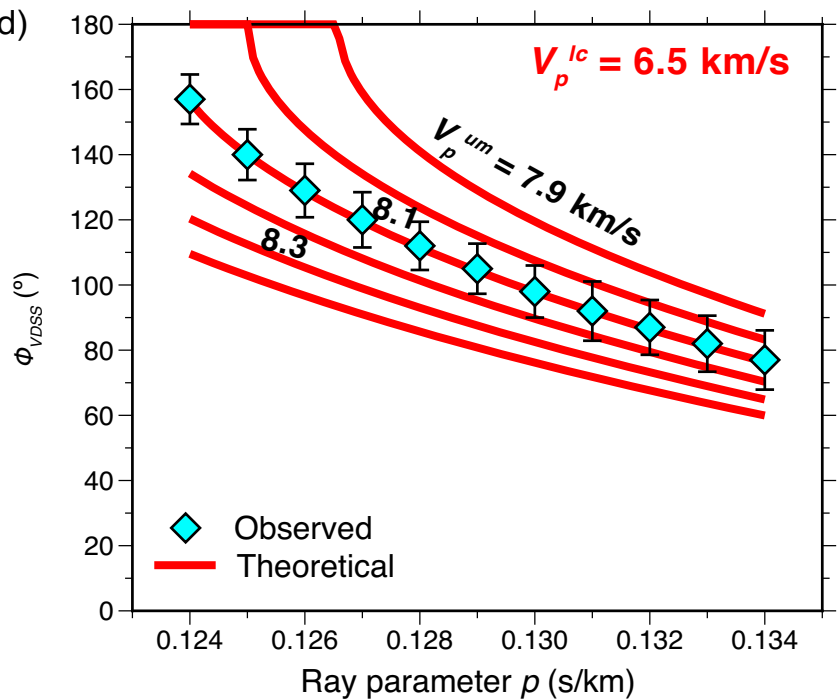

Figure 5. Synthetic example of constraining crustal and upper mantle properties with SsPmp of multiple events observed at a single station. (a) $P$ and $S$-component synthetic waveforms for events with $0.124 \leq p \leq 0.134 \mathrm{~s} \mathrm{~km}^{-1}$. Black arrow marks the event shown in (b). Note that $T_{\mathrm{VDSS}}$ decreases with increasing $p$. (b) Observed and best-fitting $P$-component waveforms for the event with $p=0.126 \mathrm{~s} \mathrm{~km}^{-1}$. (c) Observed and best-fit $T_{\mathrm{VDSs}}$ as functions of ray parameter from linear regression (Kang et al. 2016). Note $V_{p}{ }^{\text {av }}$ and $H$ are both recovered well. (d) Observed and theoretical relations between $\Phi_{\mathrm{VDSS}}$ and ray parameter for variable $V_{p}^{\text {um }}$ but $V_{p}^{\text {lc }}$ fixed at $6.5 \mathrm{~km} \mathrm{~s}^{-1}$. Note $V_{p}^{\text {um }}$ is well-recovered.

We also test our multi-event method using noisy synthetic data. We contaminate our synthetic data with the same 10 per cent bandlimited white noise as described before (Fig. S4a). Whereas $\mathrm{Ss}_{S} \mathrm{Pm} p$ is clearly observed despite the addition of noise, $S p$, the phase used in $S$ receiver functions, is barely observable (Fig. S4a). We then process the noisy synthetic waveforms as described above. The measured $T_{\text {VDSS }}$ and $\Phi_{\text {VDSS }}$ have significantly higher uncertainties compared to the noise-free case $\left(0.2 \mathrm{~s}\right.$ versus $0.1 \mathrm{~s}$ and $14^{\circ}$ versus $8^{\circ}$ for the event with $p=0.126 \mathrm{~s} \mathrm{~km}^{-1}$; Fig. S4b). Nonetheless, our method still yields $V_{p}$ av and $H$ that agree reasonably well with the input model $\left(6.4 \pm 0.2 \mathrm{~km} \mathrm{~s}^{-1}\right.$ versus $6.5 \mathrm{~km} \mathrm{~s}^{-1}$ and $38 \pm 3 \mathrm{~km}$ versus $40 \mathrm{~km}$; Fig. S4c). The observed $\Phi_{\mathrm{VDSs}}-p$ relation also favours $V_{p}$ um $=8.1 \pm 0.1 \mathrm{~km} \mathrm{~s}^{-1}$ despite scattering of the data points (Fig. S4d). In short, we show that our proposed multievent analysis can robustly retrieve $V_{p}^{\text {av }}, H$ and $V_{p}^{\text {um }}$ from data with moderate noise levels, making it suitable for application to field data.

\subsection{Average crustal $V_{p} / V_{s}$ ratio $(\kappa)$ and composition from joint PRF-VDSS analysis}

Because rock $V_{p} / V_{s}$ ratios are sensitive to rock composition (Christensen 1996), estimation of average crustal $V_{p} / V_{s}$ ratio (also denoted $\kappa)$ can offer insights into bulk composition of the crust, thus tectonic evolution of the area. Conventionally, $\kappa$ has been estimated using the $H-\kappa$ method, which searches for the model that maximizes the stacked energy of Moho Ps and multiples on PRFs (PpPms, PpSms, etc.; Zhu \& Kanamori 2000). However, the conventional $H-\kappa$ method has two major disadvantages. First, it uses the Moho multiples on PRF, which are not always robustly observed. Secondly, 


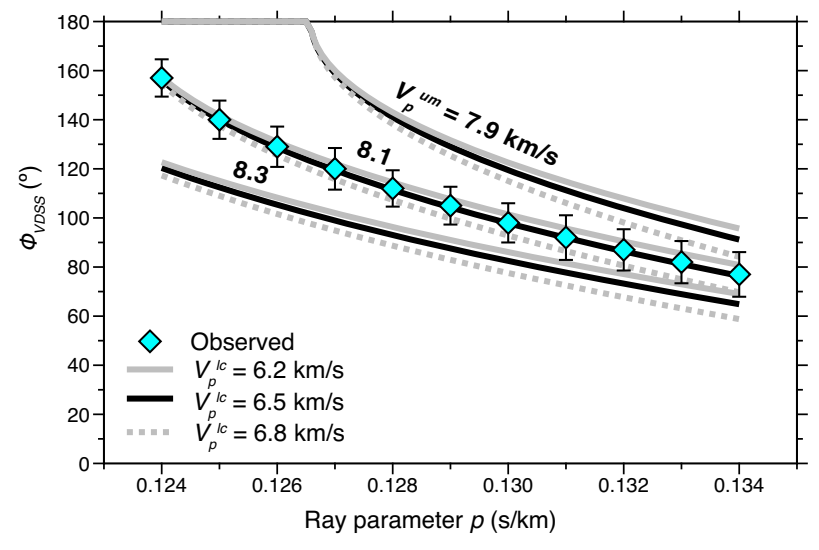

Figure 6. Effects of assumed $V_{p}{ }^{\text {lc }}$ on $V_{p}$ um derived from observed $\Phi_{\text {VDSs }}$ as a function of ray parameter. The black curves $\left(V_{p}{ }^{\mathrm{lc}}=6.5 \mathrm{~km} \mathrm{~s}^{-1}\right)$ and data points are the same as the ones in Fig. 5(d).

it requires $V_{p}$ av as an input parameter, which needs to be estimated independently. A recent proposal to complement conventional $H-\kappa$ method with SsPmp observations in order to estimate $V_{p}{ }^{\text {av }}$ still requires robust observation of at least one Moho multiples on PRFs (Luo et al. 2018). Here, we present a joint PRF-VDSS analysis method to derive $\kappa$ without this limitation by using the moveout of SsPmp to constrain $V_{p}$ av and $H$.

With a 1-D assumption, the arrival time of the Moho Ps in PRF can be expressed as:

$T_{P s}=H\left(\sqrt{\left(\frac{k}{V_{p}^{a v}}\right)^{2}-p^{2}}-\sqrt{\left(\frac{1}{V_{p}^{a v}}\right)^{2}-p^{2}}\right)$

Using $H$ (hereafter $H_{\mathrm{VDSS}}$ ) and $V_{p}^{\text {av }}$ given by $S s P m p$ observations, we can derive $\kappa$ (hereafter $\kappa_{\text {joint }}$ ) from $T_{P S}$ (Fig. 7). To estimate the uncertainty of our $\kappa_{\text {joint }}$, we draw 5000 random samples of $V_{p}{ }^{\text {av }}$ and $H_{\mathrm{VDSS}}$ from their joint distribution (Kang et al. 2016) and compute $\kappa_{\text {joint }}$ for each pair. We then estimate uncertainty of our $\kappa_{\text {joint }}$ from the variance of the 5000 randomly simulated $\kappa_{\text {joint }}$ (Fig. 7). Since $V_{p}{ }^{\text {av }}$ and $\kappa_{\text {joint }}$ together place key constraints on average crustal composition (Christensen \& Mooney 1995; Christensen 1996), we also compute the joint distribution of $V_{p}$ av and $\kappa_{\text {joint }}$ and compare them with laboratory measurements (Fig. 8).

We again use synthetic data to test our method. We generate synthetic PRFs using Model \#1, apply normal moveout, and stack them. From the stacked PRF, the Moho $T_{P_{S}}$ is picked at $4.50 \mathrm{~s}$ (Fig. 7a). Using our $T_{P_{s}}$ and the previously estimated $V_{p}{ }^{\text {av }}=6.5 \pm 0.1 \mathrm{~km} \mathrm{~s}^{-1}$ and $H_{\mathrm{VDSS}}=40 \pm 1 \mathrm{~km}$, we find $\kappa_{\text {joint }}=1.73 \pm 0.02$ (Fig. $7 \mathrm{~b}$ ), consistent with the input model. We also plot the joint distribution of $V_{p}{ }^{\text {av }}$ and $\kappa_{\text {joint }}$, which shows a clear negative correlation between the two parameters (Fig. 8). To compare our seismic 'observations' with laboratory measurements, we plot $V_{p}$ and $\kappa$ of major crustal rock types measured at $600 \mathrm{MPa}$ (corresponds to mid-crustal depth) and room temperature from Christensen (1996, Fig. 8). We also plot $V_{p}, \kappa$ and $\mathrm{SiO}_{2}$ content of individual samples (excluding eclogites and ultramafic rocks) measured at $600 \mathrm{MPa}$ and room temperature (compilation of Hacker et al. 2015). For typical crustal rocks, $\mathrm{d} V_{p} / \mathrm{d} T=\sim-0.4 \mathrm{~m} \mathrm{~s}^{-1}{ }^{\circ} \mathrm{C}^{-1}$, and $\mathrm{d} V_{s} / \mathrm{d} T=\sim-0.2 \mathrm{~m} \mathrm{~s}^{-1}{ }^{\circ} \mathrm{C}^{-1}$, so typical $\mathrm{d} \kappa / \mathrm{d} T=\sim-0.0015 / 100{ }^{\circ} \mathrm{C}$ (Christensen \& Mooney 1995; Barruol \& Kern 1996; Christensen 1996). With these relations, we apply temperature corrections to the measurements from Christensen (1996) and Hacker et al. (2015) assuming a mid-crustal temperature of $\sim 250^{\circ}$, suitable for cratonic crust. We note that the temperature correction $\left(-0.1 \mathrm{~km} \mathrm{~s}^{-1}\right)$ is moderate for $V_{p}$, but negligible $(-0.004)$ for $\kappa$, indicating that $\kappa$ is primarily controlled by rock composition (Christensen 1996). The laboratory measurements show a clear correlation of increasing $V_{p}$ and $\kappa$ with decreasing $\mathrm{SiO}_{2}$ content (Fig. 8). Because our $V_{p}-\kappa$ correlation from VDSS-PRF analysis is orthogonal to the $V_{p}-\kappa$ correlation of laboratory measurements, our method constrains the average crustal composition in this synthetic example as intermediate (equivalent to diorite or felsic granulite, Fig. 8). We recognize the large scatter of individual laboratory measurements of similar composition (coloured circles; Hacker et al. 2015) and the large variation between aggregated samples that share the same rock name (diamonds; Christensen 1996) around the positive $V_{p}-\kappa$ trend, but note that these compilations represent samples collected in highly diverse geological settings. When applying our method to a specific region, a more desirable approach would be to build a rock physics model appropriate for that region.

\section{OBSERVATIONS AND INTERPRETATIONS OF THE SOUTHWEST SLAVE CRATON}

The Canadian Shield has a longevity and stability suggestive of a lithospheric structure closer to 1-D than tectonically active areas. The Yellowknife Array was deployed in the Slave Craton of the Canadian Shield (Figs 9a and b) in 1962 and was upgraded to digital recording in 1989 (Bostock 1998). The long deployment time and its location at the core of the Canadian Shield makes it an ideal place to test our proposed method for retrieving and analysing both $T_{\text {VDSS }}$ and $\Phi_{\text {VDSs }}$ under a 1-D assumption. In addition, the LITHOPROBE project acquired wide-angle refraction and near-vertical reflection profiles through Yellowknife (Fernández-Viejo \& Clowes 2003; Fernández-Viejo et al. 2005; Hammer et al. 2010), allowing direct comparison between VDSS and active-source results.

\subsection{VDSS and PRF data analysis}

Among stations of the Yellowknife Array, YKW3 has the longest archived broadband recording (1994-2014). In addition, Station EDZN of the POLARIS array (Snyder \& Bruneton 2007) was located $\sim 80 \mathrm{~km} \mathrm{NW}$ of YKW3, within $\sim 25 \mathrm{~km}$ of SsPmp Moho reflection points for Kamchatka-Kurile earthquakes recorded at YKW3 (Fig. 9b), allowing us to compare PRF results at EDZN with VDSS results at YKW3, as conversion points of PRFs at Moho depth are typically $<20 \mathrm{~km}$ away from the station. For VDSS analysis at YKW3, we examined 56 teleseismic events in the epicentral range $30-60^{\circ}$ with the back-azimuth range of $290-310^{\circ}$. We choose this narrow backazimuth range to avoid possible complications from lateral variation in lithospheric structure [the Moho reflection points of our selected events are all within $25 \mathrm{~km}$ of each other (Fig. 9b)], while still including plenty of events from the Kamchatka subduction zone with a wide range of ray parameters (Figs 9a and c). We removed instrumental response and applied a bandpass Butterworth filter between 0.05 and $0.5 \mathrm{~Hz}$. After separating radial and vertical-component data into $P$ and $S$ components, we inspected the traces and their particle-motion diagrams, selecting the 19 events with simple $S s$ waveforms and significant $P$ energy following the $S$ arrival. We next computed the envelope functions of the $P$ and $S$ components of the 19 events and rejected the nine 'Grade C' events for which the ratio of the maxima of the $P$ and $S$ envelope functions $\leq 0.6$. The remaining 10 events all have simple $S s$ waveforms and 

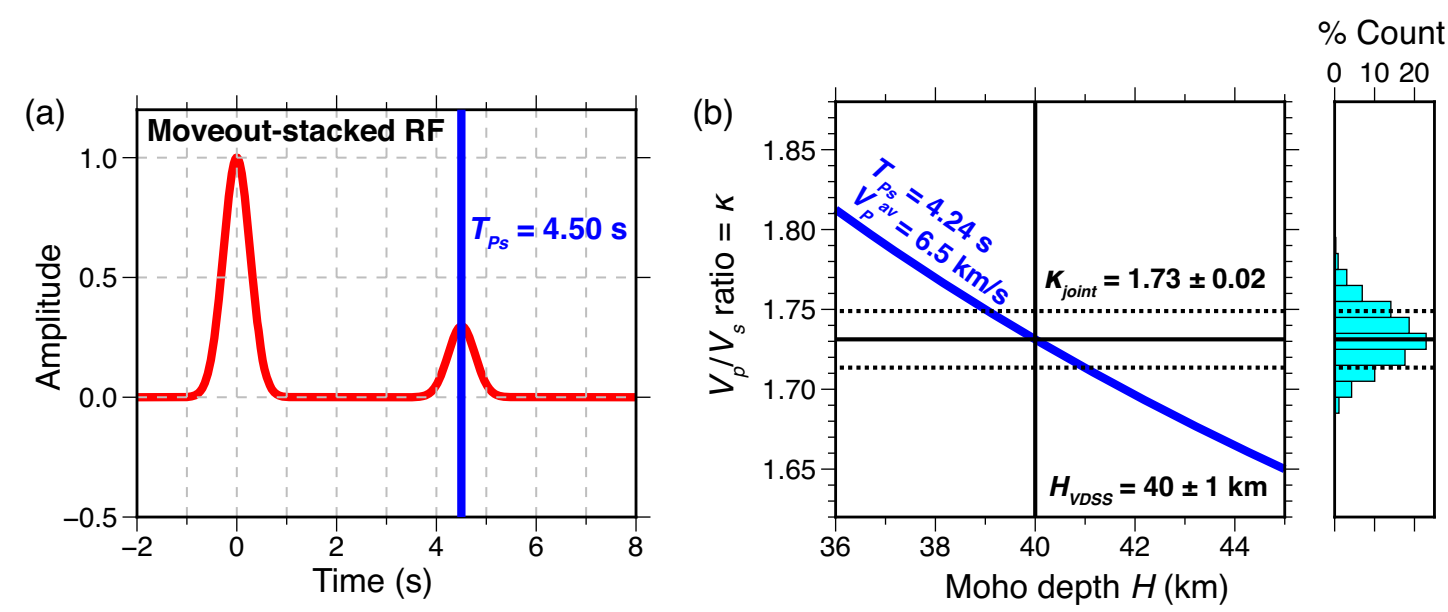

Figure 7. Synthetic example of constraining average crustal $V_{p} / V_{S}$ ratio $(\kappa)$ via PRF-VDSS joint analysis. (a) Synthetic PRF computed with Model \#1, after moveout-correction to normal incidence. (b) Determination of crustal average $V_{p} / V_{s}$ ratio $\left(\kappa_{\text {joint }}\right)$ from PRF $T_{P s}$, VDSS Moho depth $\left(H_{\mathrm{VDSS}}\right)$, and VDSS average crustal $V_{p}\left(V_{p}{ }^{\text {av }}\right)$. Dashed black lines mark the estimated uncertainty of $\kappa_{\text {joint }}$, which is computed by taking the standard deviation of 5000 random simulations of $\kappa_{\text {joint }}$ (cyan histogram). Note $\kappa$ is well recovered.

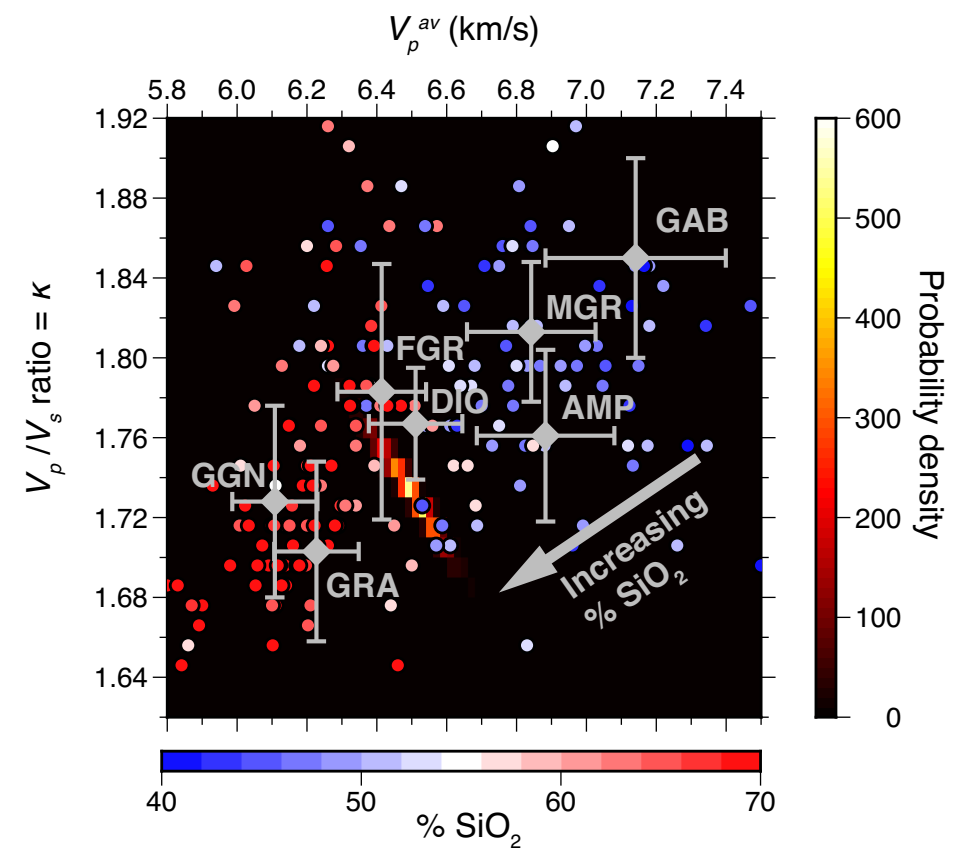

Figure 8. Synthetic example of constraining average crustal composition with PRF-VDSS joint analysis. The probability density function is derived from 5000 random simulations of $V_{p}$ av and $\kappa$. Circles coloured by per cent $\mathrm{SiO}_{2}$ are measurements for crustal rock samples (felsic to mafic) at $600 \mathrm{MPa}$ and room temperature (compilation of Hacker et al. 2015), which are then corrected to $250{ }^{\circ} \mathrm{C}$ (see Section 2.4). Gray diamonds and data ranges are measurements at $600 \mathrm{MPa}$ and room temperature from Christensen (1996), which are also corrected to $250{ }^{\circ} \mathrm{C}$. GAB: Gabbro-norite-troctolite; MGR: Mafic granulite; DIO: Diorite; FGR: Felsic granulite; GGN: Granite gneiss; GRA: Granite-granodiorite; AMP: Amphibolite. Note the orthogonal trends of the probability density function and per cent $\mathrm{SiO}_{2}$, which constrains average crustal composition.

strong $S s P m p$ following the $S s$ arrival (Fig. 9c). When sorted by their ray parameter, the 10 traces show a clear decrease of $T_{\text {VDSS }}$ (moveout) with increasing ray parameter (Fig. 9c). To measure $T_{\mathrm{VDSS}}$ and $\Phi_{\text {VDSS }}$, we used a 20 s-window around $S s$ as our source wavelet to account for the slightly lower frequency of the observed data compared with our synthetics. After measuring $T_{\mathrm{VDSS}}$ and $\Phi_{\mathrm{VDSS}}$, we excluded three 'Grade B' events with minimum misfit $>0.4$, because their large misfit implies that $S s P m p$ is poorly approximated by a phase-shifted source time function, violating a basic assumption. We used measured $T_{\text {VDSS }}$ and $\Phi_{\text {VDSS }}$ of the remaining seven
'Grade A' to derive $V_{p}^{\text {av }}=6.7 \pm 0.2 \mathrm{~km} \mathrm{~s}^{-1}, H=38 \pm 4 \mathrm{~km}$ (Fig. 9e) and $V_{p}^{\text {um }}=\sim 8.0-8.1 \mathrm{~km} \mathrm{~s}^{-1}$ (Fig. 9f) as described above.

For PRF analysis at EDZN, we used a time-domain iterative deconvolution algorithm with a Gaussian bandwidth of $2.5 \mathrm{~Hz}$ (Herrmann 2013) to compute PRFs for teleseismic $P$-wave data from $30-90^{\circ}$ (Fig. S5). We manually selected 73 high-quality radial RFs using the Funclab software package (Porritt \& Miller 2018), moveout-corrected the RFs to normal incidence for primary $P S$ conversions and stacked, which yields a clear Moho $P s$ at $4.24 \mathrm{~s}$ and little energy from intra-crustal converters (Fig. 10a). Using 
(a)

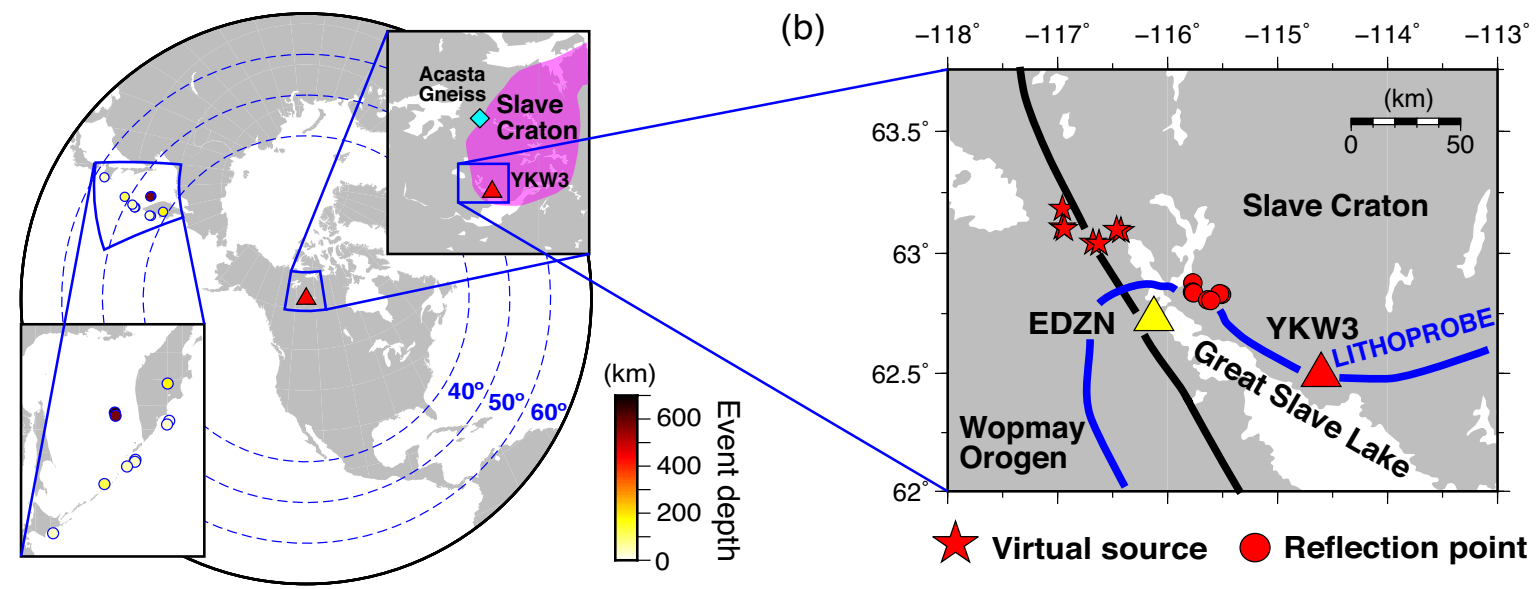

(c)

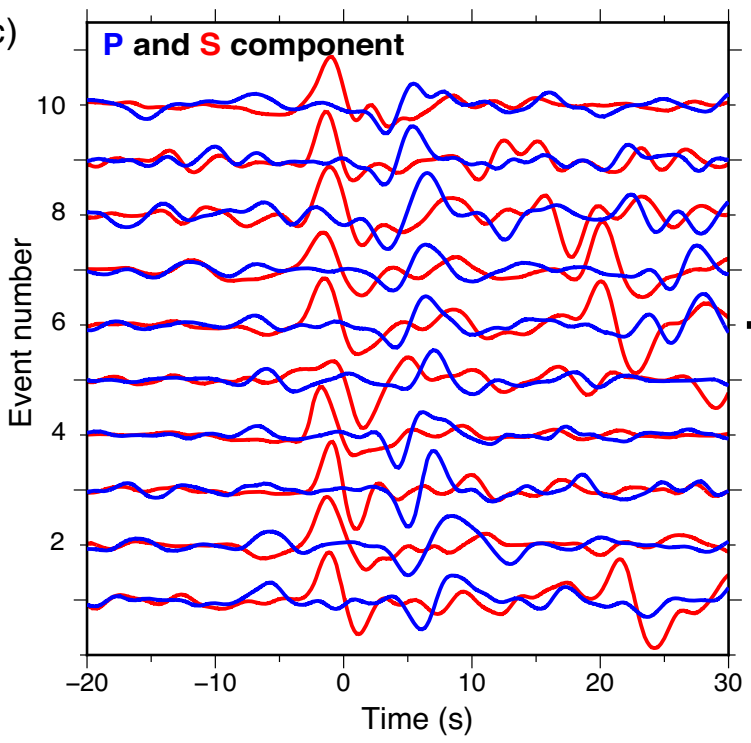

(e)

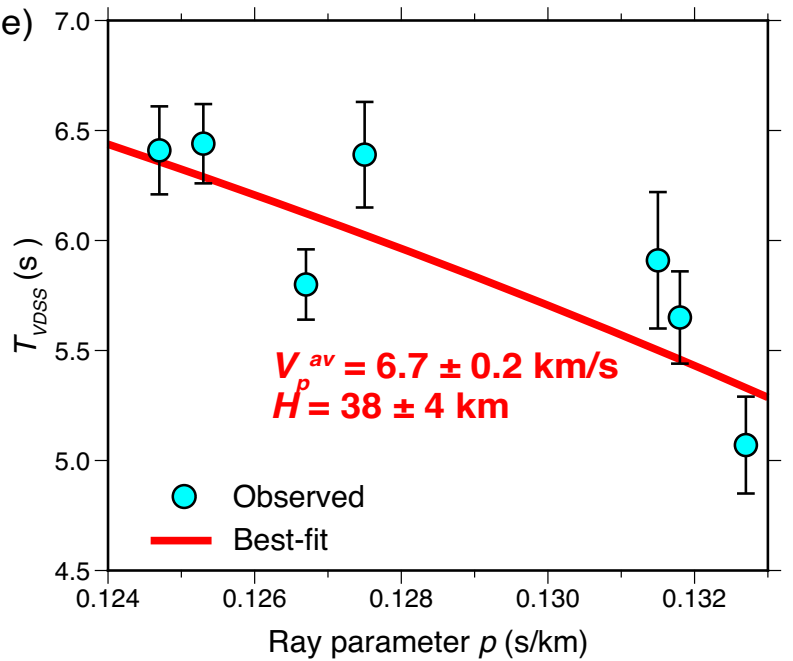

(d)

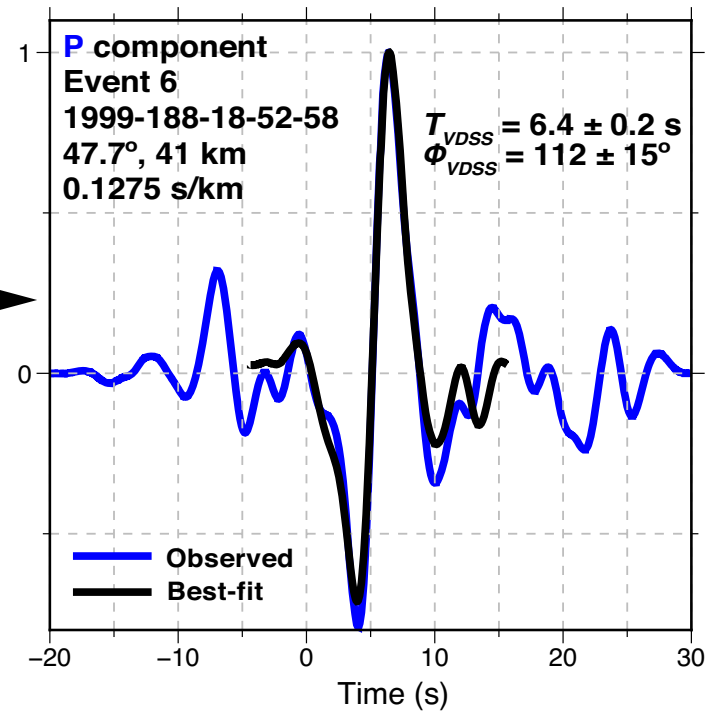

(f)

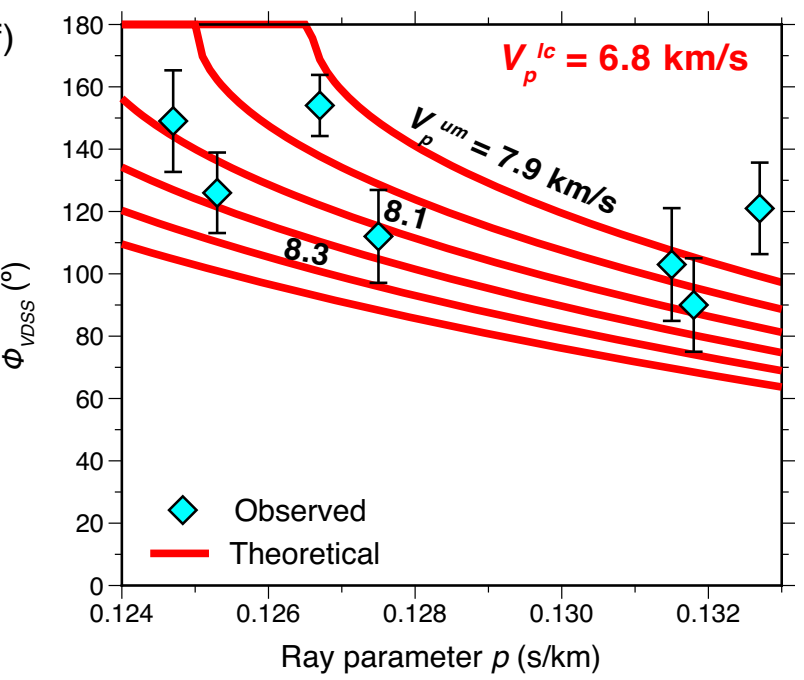

Figure 9. $V_{p}^{\text {um }}, V_{p}$ av and Moho depth $H$ near station YKW3 derived from $T_{\mathrm{VDSS}}$ and $\Phi_{\mathrm{VDSS}}$. (a) Station and event distribution on a polar plot centered on YKW3. 10 Grade A and B events with backazimuth $297-304^{\circ}, M_{\mathrm{W}}>5.5$, distance $40-60^{\circ}$ and depth $30-700 \mathrm{~km}$, are coloured by their focal depth. (b) Locations of broadband stations YKW3 and EDZN (triangles), LITHOPROBE reflection and refraction profiles (blue line), and VDSS Moho reflection points and virtual sources. The reflection-point and virtual-source locations are computed using the best-fitting $V_{p}{ }^{\text {av }}$ and $H$ (part e) for the seven Grade A events (part c). PRF results for EDZN are shown in Figs. 10 and S5. (c) $P$ - and $S$-component waveforms of the ten Grade A and B events. Events are sorted by their ray parameters (small to large), with detail information in Table S1. Black arrow marks Event 6 shown in (d). (d) Observed and best-fitting $P$-component waveforms of Event 6. (e) Observed $T_{\mathrm{VDSS}}$ as functions of ray parameter and the best-fitting model from linear regression. (f) Observed and theoretical relations between

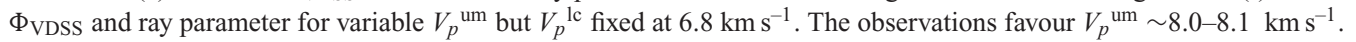



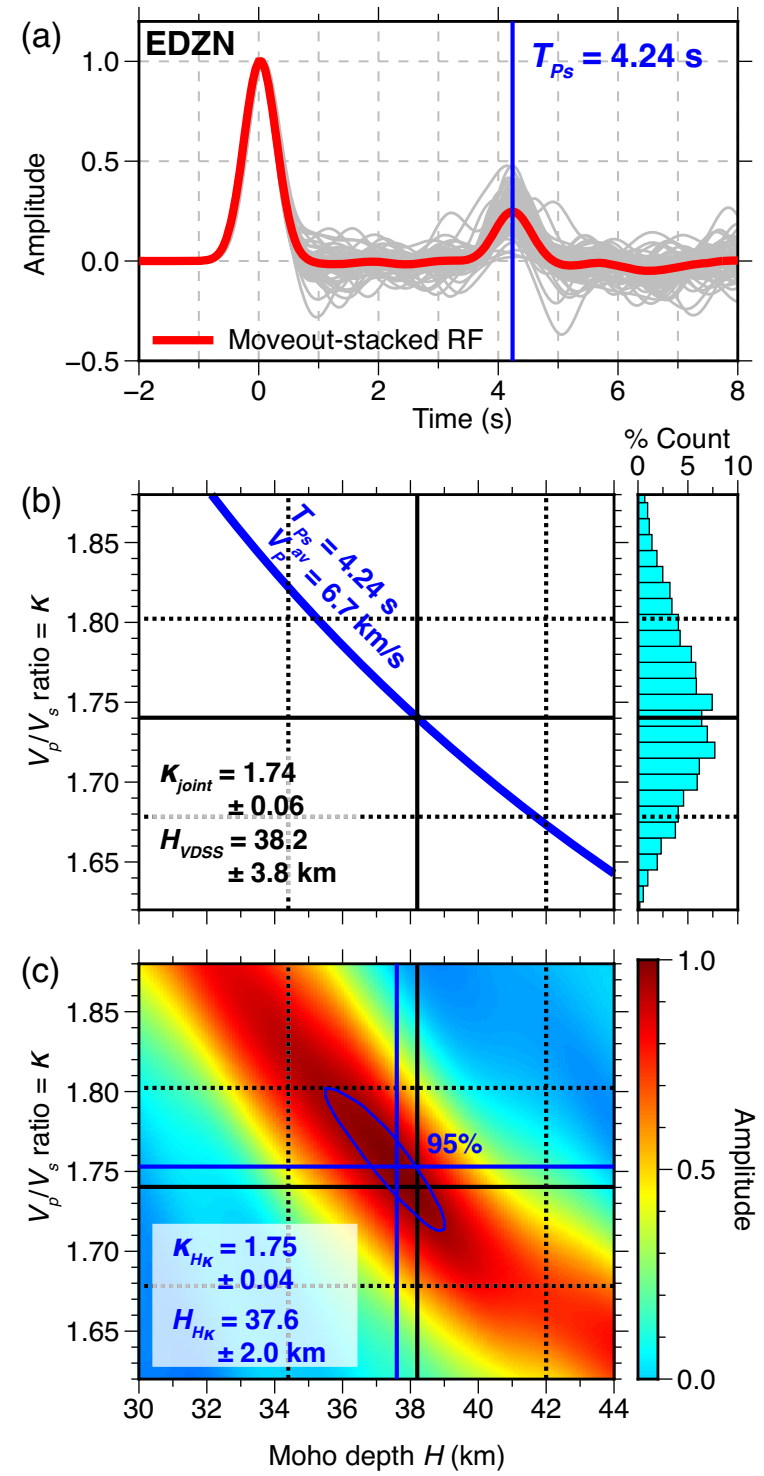

Figure 10. PRF-VDSS joint analysis for crustal structure of the SW Slave Craton. (a) PRF waveforms of EDZN. The stacked PRF is computed by summing 73 PRFs (grey curves) moveout-corrected to normal incidence. (b) Determination of average crustal $V_{p} / V_{s}$ ratio $\left(\kappa_{\text {joint }}\right)$ from PRF $T_{P s}$, VDSS Moho depth $\left(H_{\mathrm{VDSs}}\right)$, and average crustal $V_{p}\left(V_{p}{ }^{\text {av }}\right)$. The dashed black lines mark the uncertainties of $\kappa_{\text {joint }}$, which is computed by taking the standard deviation of 5000 random simulations of $\kappa_{\text {joint }}$ (cyan histogram). (c) Comparison between $H_{\mathrm{VDSS}}$ and $\kappa_{\text {joint }}$ and $H$ and $\kappa$ derived from conventional $H-\kappa$ stacking $\left(H_{H \kappa}\right.$ and $\left.\kappa_{H \kappa}\right)$. Background colour is normalized amplitude of the conventional $H-\kappa$ stack computed with $V_{p}{ }^{\text {av }}=6.7 \mathrm{~km} \mathrm{~s}^{-1}$ and phase weight ratios $w_{P s}: w_{P p P m s}: w_{P p S m s}=1: 0.5: 0.5$. The $>95$ per cent amplitude range is outlined in blue, from which the uncertainty of $H_{H \kappa}$ and $\kappa_{H \kappa}$ are estimated. Note $H$ and $\kappa$ derived with the two methods are very close $(<1$ per cent difference), whereas the uncertainties are on the same order of magnitude.

$T_{P s}$ measured from our moveout-stacked PRFs and $H$ and $V_{p}$ av from our VDSS analysis, we estimate $\kappa_{\text {joint }}=1.74 \pm 0.06$ (Fig. 10b). We also performed conventional $H-\kappa$ stacking of our 73 selected RFs using a phase weight ratio $w_{P s}: w_{P p P m s}: w_{P p S m s}=1: 0.5: 0.5(\mathrm{Zhu}$ \& Kanamori 2000) and $V_{p}{ }^{\text {av }}=6.7 \mathrm{~km} \mathrm{~s}^{-1}$ taken from our VDSS analysis to determine $H_{H \kappa}=37.6 \mathrm{~km}$ and $\kappa_{H \kappa}=1.75$ (Fig. 10c). We estimate the uncertainties of $H_{H \kappa}$ and $\kappa_{H \kappa}( \pm 2.0 \mathrm{~km}$ and \pm 0.04 ,

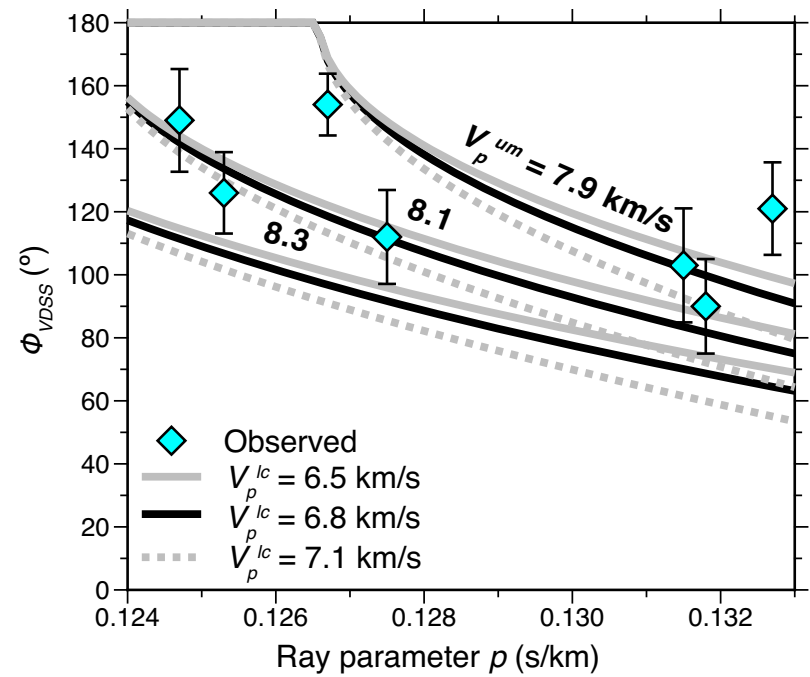

Figure 11. Effects of assumed $V_{p}^{\text {lc }}$ on $V_{p}$ um derived from observed $\Phi_{\text {VDSS }}$ as a function of ray parameter at YKW3. The black curves $\left(V_{p}^{\mathrm{lc}}=6.8 \mathrm{~km} \mathrm{~s}^{-1}\right)$ and observations (data points) are the same as the ones in Fig. 9(f).

respectively) using the range with stacking amplitude $>95$ per cent of the peak value while ignoring the additional uncertainties arising from the choice of $V_{p}{ }^{\text {av }}$ and phase stacking weights (supplementary figures in Karplus et al. 2019).

We also computed transverse PRFs. We found neither azimuthal variation of Moho PS on the radial PRFs (Figs S5b and c) nor clear Moho Ps on the transverse PRFs (Figs S5d and e), indicating negligible Moho dip or crustal anisotropy. We do however observe a coherent negative arrival at $\sim 8 \mathrm{~s}$ on the transverse PRFs (Figs $\mathrm{S} 5 \mathrm{~d}$ and e), which may correspond to the intra-mantle 'H converter' imaged beneath Yellowknife by Bostock (1998).

\subsection{Uncertainties and sensitivities of VDSS analyses}

The observed $T_{\mathrm{VDSS}}$ of Grade A events clearly decreases with increasing ray parameter, with a typical uncertainty of $\pm 0.3 \mathrm{~s}$ (Fig. 9e). We find the best-fitting crustal $V_{p}{ }^{\text {av }}$ and Moho depth ( $\left.H_{\text {VDSs }}\right)$ to be $6.7 \pm 0.2 \mathrm{~km} \mathrm{~s}^{-1}$ and $38 \pm 4 \mathrm{~km}$, respectively (Fig. 9e). The observed $\Phi_{\text {VDSs }}$ of Grade A events also shows a clear decrease with increasing ray parameter (Fig. 9f), in accord with our theoretical predictions (Part. 1). To find the best-fitting $V_{p}$ um , we plot theoretical $\Phi_{\text {VDSs }}-p$ relations for different $V_{p}$ um values while fixing $V_{p}^{\text {lc }}=6.8 \mathrm{~km} \mathrm{~s}^{-1}$, the value given by wide-angle refraction studies close to our VDSS reflection points (Fig. 9f, Fernández-Viejo \& Clowes 2003). Comparing our observed values with the theoretical relations, we find that the observations favour $V_{p}^{\text {um }}=8.0-8.1 \mathrm{~km} \mathrm{~s}^{-1}$, consistent with the $P n$ velocity of $8.2 \pm 0.1-0.2 \mathrm{~km} \mathrm{~s}^{-1}$ reported by the wide-angle refraction studies (Fernández-Viejo \& Clowes 2003; FernándezViejo et al. 2005). Our choice of $V_{p}^{\text {lc }}$ has little effect on estimated $V_{p}{ }^{\text {um }}$ given the $\sim 20^{\circ}$ uncertainty in observed $\Phi_{\mathrm{VDSS}}$, as shown by theoretical curves computed assuming $V_{p}^{\text {lc }}=6.5,6.8$ and $7.1 \mathrm{~km} \mathrm{~s}^{-1}$ ( $\sim 4$ per cent perturbation) (Fig. 11). This is because the range of $1 / p$ considered here $\left(7.5-8.0 \mathrm{~km} \mathrm{~s}^{-1}\right)$ is significantly higher than the assumed $V_{p}$ lc , making $\Phi_{\mathrm{VDSS}}$ insensitive to $V_{p}{ }^{\text {lc }}$ (Fig. 7 in Part 1).

In addition to using only our seven Grade $\mathrm{A}$ events, we test the effects of also including Grade $\mathrm{B}$ and $\mathrm{C}$ events, which gives significantly higher values for $V_{p}{ }^{\text {av }}$ and $H$ (Fig. S6a). This increase in $V_{p}$ av and $H$ is primarily due to the large $T_{\mathrm{VDSs}}$ of Grade $\mathrm{B}$ and $\mathrm{C}$ events with small ray parameter (Fig. S6a). Similarly, inclusion of 
Grade B and C events significantly increases our estimate of $V_{p}$ um (Fig. S6b). This increase in $V_{p}^{\text {av }}, H$ and $V_{p}^{\text {um }}$ is primarily due to most of the Grade B and $\mathrm{C}$ events coincidentally having smaller ray parameters than Grade A events, even though our data selection was not based on ray parameter. For events with $p<0.122 \mathrm{~s} \mathrm{~km}^{-1}$, the turning velocity $1 / p$ of the downgoing $P$ wave exceeds $8.2 \mathrm{~km} \mathrm{~s}^{-1}$, higher than the velocity we estimated for the top of the upper mantle $\left(V_{p}^{\text {um }}=8.1-8.0 \mathrm{~km} \mathrm{~s}^{-1}\right)$. Therefore, SsPmp with $p<0.122 \mathrm{~s}$ $\mathrm{km}^{-1}$ has turned or been refracted within the upper mantle below the CMB. These events thus likely provide estimates of $V_{p}^{\text {um }}$ more appropriate for the depth at which the rays turn, and estimates of $V_{p}{ }^{\text {av }}$ and $H$ that average the crust and upper mantle. Previous wide-angle refraction results suggested that $V_{p}$ increases from $8.2 \mathrm{~km} \mathrm{~s}^{-1}$ right below the Moho to $8.5 \mathrm{~km} \mathrm{~s}^{-1}$ at $\sim 65 \mathrm{~km}$ depth between YKW3 and EDZN (Fernández-Viejo \& Clowes 2003), consistent with our observation that $\Phi_{\mathrm{VDSS}}$ at low ray parameter suggests $V_{p}^{\text {um }}=8.4$ $8.6 \mathrm{~km} \mathrm{~s}^{-1}$ (Fig. S6b). Additional difficulties may arise if the turning velocity is only slightly higher than the velocity just below a sharp $\mathrm{CMB}$, because strong pre-critical reflections at the CMB will interfere with $S s P m p$ that turns in the upper mantle (Fig. 5 in Part 1), causing a complicated $S s P m p$ waveform that cannot be modeled with a phase-shifted $S$ wavelet. Moreover, as $p$ decreases and SsPmp becomes a turning wave in the upper mantle, the distance between the virtual source and station grows, increasing the effects of lateral heterogeneity on $T_{\text {VDSS }}$ and $\Phi_{\text {VDSs }}$. These complications may cause the large uncertainty and scatter of measured $T_{\mathrm{VDSS}}$ and $\Phi_{\text {VDSs }}$ for events with small $p$ (Fig. S6). In summary, when constraining $V_{p}{ }^{\text {av }}$ and $H$ with $T_{\mathrm{VDSS}}$, it is appropriate to only use events with $1 / p$ smaller than $V_{p}$ um, because the behaviour of $S s P m p$ as an upper-mantle turning wave is more complicated than modelled here. Nonetheless, this behaviour may open the way to continuous depth profiling of upper-mantle velocity structure in future work.

\subsection{Comparison with previous imaging and geologic interpretations}

Our Moho depth $\left(H_{\text {VDSS }}=38 \pm 4 \mathrm{~km}\right.$, Fig. $\left.9 \mathrm{e}\right)$ is in good agreement with LITHOPROBE near-vertical reflection results $(\sim 37 \mathrm{~km})$, but slightly deeper than their seismic refraction results $(\sim 31-33 \mathrm{~km}$, Fernández-Viejo et al. 2005; Hammer et al. 2010, Fig. 12). Our average crustal $V_{p}\left(V_{p}{ }^{\text {av }}=6.7 \pm 0.2 \mathrm{~km} \mathrm{~s}^{-1}\right.$, Fig. 9e $)$ is also higher than the value given by the wide-angle refraction studies $\left(\sim 6.4 \mathrm{~km} \mathrm{~s}^{-1}\right.$, Fernández-Viejo et al. 2005; Hammer et al. 2010, Fig. 12). Our $H_{\mathrm{VDSS}}$ closely matches our $H-\kappa$ Moho depth $\left(H_{\mathrm{VDSS}}=38.2 \pm 3.8 \mathrm{~km} ; H_{H \kappa}=37.6 \pm 2.0 \mathrm{~km}\right.$; Fig. 10c $)$. This agreement not only verifies our VDSS method, but also indicates that $V_{p}$ av $=6.7 \mathrm{~km} \mathrm{~s}^{-1}$ assumed for the $H-\kappa$ analysis is likely a reliable estimation of average crustal $V_{p}$. Similarly, the agreement between $\kappa_{\text {joint }}=1.74 \pm 0.06$ and $\kappa_{H \kappa}=1.75 \pm 0.04$ (Fig. 10) further demonstrates the robustness of our method, though our $\kappa$ values are higher than those from the refraction study that found $\kappa=1.68-1.72$ in the Slave Craton (Fernández-Viejo et al. 2005).

Our joint distribution between $V_{p}{ }^{\text {av }}$ and $\kappa_{\text {joint }}$ shows a clear negative correlation (Fig. 13), similar to our synthetic example (Fig. 8), though the field data is less focused than the synthetics due to larger observation uncertainty. We again compare the joint distribution with the temperature-corrected laboratory measurements from Christensen (1996) and Hacker et al. (2015), which implies an intermediate (dioritic) average crustal composition, though some mafic rock samples also fit our preferred $V_{p}{ }^{\text {av }}-\kappa$ estimates (Fig. 13). The refraction $V_{p}$ av and $\kappa$ values (Fernández-Viejo et al. 2005) are quite distinct (green ellipse in Fig. 13) and imply a felsic (granitic) average crustal composition.

Fernández-Viejo et al. (2005) interpreted their low average $V_{p}$ av and $\kappa$ as evidence for a 'significant felsic content' of the crust in the SW Slave Craton and explained the regional Bouguer gravity low with their silicic but thin crust. Our more-mafic but thicker crust could equally match the gravity signature. We also note that the lower $V_{p}$ av and thinner crust found by wide-angle refraction experiments could partly be explained by a positive trade-off between the two parameters.

The Slave craton is home to the Earth's oldest rocks, the Acasta Gneiss with Hadean ages $\geq 4$ Ga (Bowring \& Williams 1999), though the bulk of its crust is clearly Archaean. In the SW Slave Craton, geochronology of surface exposures emphasizes the importance of Neoarchaean crustal growth at $<2.6 \mathrm{Ga}$ (Bennett et al. 2005), but other crustal-scale cross-sections show the Palaeoarchean-toMesoarchean Central Slave Basement Complex forming the bulk of the crust (Bleeker 2002), as also interpreted from LITHOPROBE near-vertical reflection profiling (van der Velden \& Cook 2002). Our observation of crustal thickness, $V_{p}$ av and $\kappa$ may help resolve the dominant crustal formation age of the SW Slave Craton. A trend of increasing $V_{p}{ }^{\text {av }}, \kappa$ and crustal thickness with decreasing crustal age in the Western Australia Craton has been suggested to reflect a global transition of crust-forming mechanism from plume tectonics to plate tectonics during Archean (Yuan 2015). If this relationship were to be applicable to the Canadian cratons, our results are most consistent with a Mesoarchean formation age for the bulk crust of the SW Slave Craton.

\section{DISCUSSION}

Post-critical $S s P m p$ has three major attributes: $T_{\mathrm{VDSS}}, \Phi_{\mathrm{VDSS}}$ and $A_{\mathrm{VDSS}}$. In $1-\mathrm{D}, A_{\mathrm{VDSS}}$ is largely affected by near-surface velocity (Fig. 10 in Part 1), whereas $T_{\text {VDSs }}$ and $\Phi_{\text {VDSS }}$ both contain information on crustal and upper-mantle structure. In this paper, we propose methods to retrieve Moho depth, $V_{p}{ }^{\text {av }}$ and $V_{p}{ }^{\text {um }}$ from $S s P m p$ observations under a $1 \mathrm{D}$ assumption.

$T_{\text {VDSs }}$ is controlled by Moho depth and $V_{p}{ }^{\text {av }}$ and can be measured together with $\Phi_{\text {VDSs }}$ by waveform fitting (Fig. 5b). Whereas Moho depth can be estimated from a single SsPmp observation with an assumed $V_{p}$ av (Fig. 4), both $H_{\mathrm{VDSS}}$ and $V_{p}$ av can be constrained using multiple events recorded at the same station (Fig. 5) (Kang et al. 2016). The uncertainties of our derived $H_{\mathrm{VDSS}}$ and $V_{p}$ av at YKW3 (4 km and $0.2 \mathrm{~km} \mathrm{~s}^{-1}$, respectively) are larger than those given by Kang et al. (2016) for two stations FORT and WB2 in Australia $\left(\sim 3.0 \mathrm{~km}\right.$ and $\sim 0.15 \mathrm{~km} \mathrm{~s}^{-1}$, respectively). Two factors might contribute to the higher uncertainties of our measurements. First, the uncertainty of our $T_{\text {VDSS }}(\sim 0.3 \mathrm{~s})$ is greater than Kang et al. $(\sim 0.18 \mathrm{~s})$, but these values are not directly comparable because we compute $T_{\text {VDSs }}$ uncertainties quantitatively (Supplementary Text 1), whereas Kang et al. (2016) estimated their uncertainties empirically. Second, the ray-parameter range of our study $(0.1247-0.1327 \mathrm{~s}$ $\left.\mathrm{km}^{-1}\right)$ is smaller than in Kang et al. $\left(0.1223-0.1349 \mathrm{~s} \mathrm{~km}^{-1}\right.$ for FORT and $0.1226-0.1369 \mathrm{~s} \mathrm{~km}^{-1}$ for WB2), largely because the number of events we used (seven) is smaller than in Kang et al. (20 for FORT and 12 for WB2). Our events are fewer and our ray-parameter range smaller in part due to our strict data-selection criteria (Fig. S6). In practice, the number of observations available depends on event distribution and data quality.

$\Phi_{\text {VDSs }}$ is controlled by $V_{p}^{\text {lc }}$ and $V_{p}{ }^{\text {um }}$ and can be used to constrain the two parameters. Due to the trade-off between $V_{p}{ }^{\text {lc }}$ and 


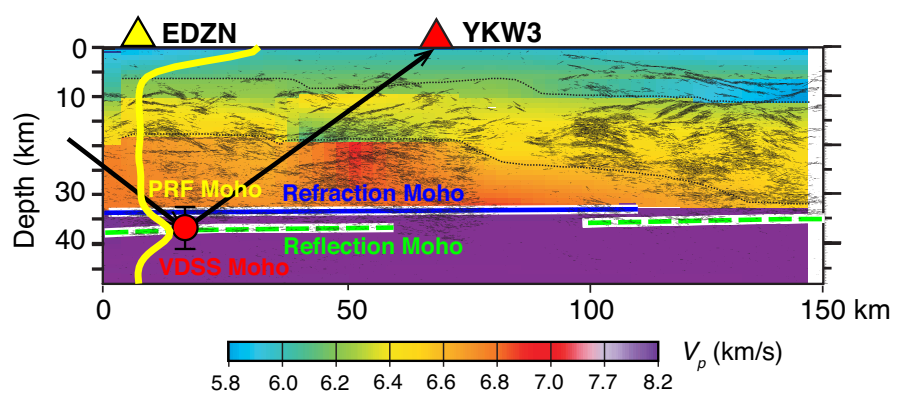

Figure 12. Comparison between our VDSS and PRF and LITHOPROBE reflection and refraction results. The migrated reflection image is overlain on the refraction $V_{p}$ model (after Hammer et al. 2010). Red circle with error bars: VDSS Moho plotted at the reflection point; the arrows are ray paths of SsPmp. Yellow curve: moveout-stacked PRF mapped to depth domain and plotted at EDZN. VDSS, PRF and reflection Moho depths agree with each other $(\sim 38 \mathrm{~km})$, but the refraction Moho is shallower ( $234 \mathrm{~km})$.

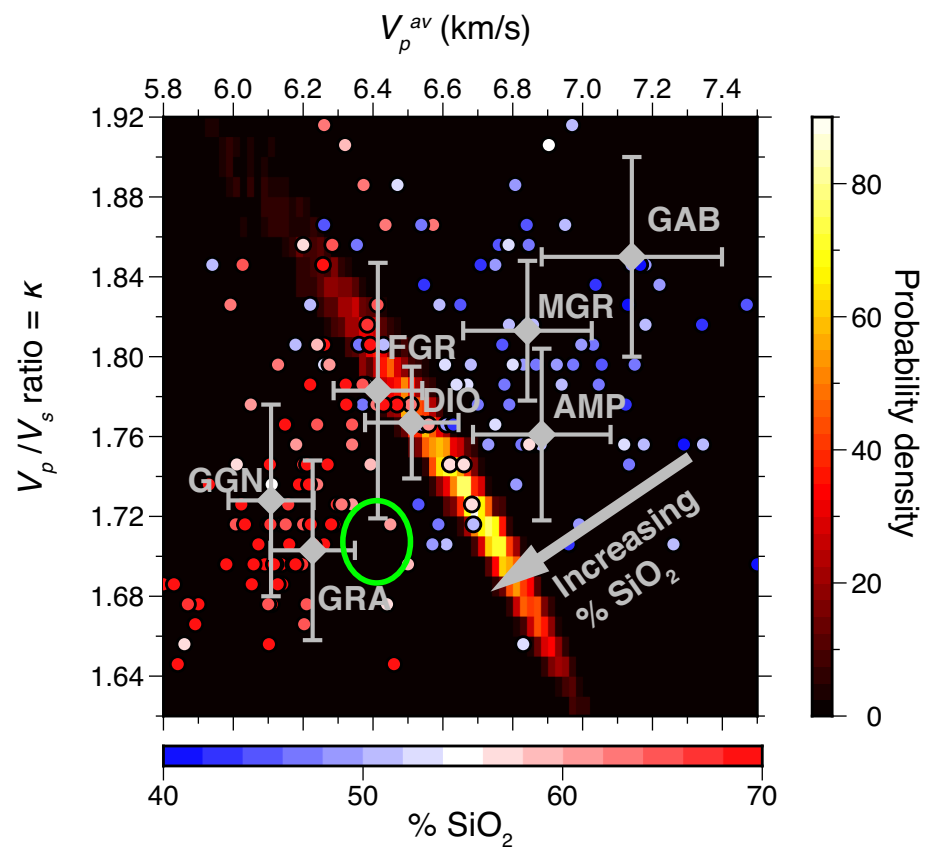

Figure 13. Average crustal composition of the SW Slave Craton constrained with PRF-VDSS joint analysis. The probability density function is derived from 5000 random simulations of $V_{p}$ av and $V_{p} / V_{s}$ ratio. The diamonds and circles are the same as Fig. 8 . The orthogonal trends of the probability density function and rock properties together constrain average crustal composition. Green ellipse is $V_{p}{ }^{\text {av }}$ and $\kappa$ from Fernández-Viejo et al. (2005).

$V_{p}^{\text {um }}$ (Fig. 7 in Part 1), it is impossible to determine one without knowing the other (Fig. 3). However, we show that when $p$ is small $\left(1 / p\right.$ close to $\left.V_{p}^{\text {um }}\right), \Phi_{\text {VDSs }}$ depends only weakly on $V_{p}^{\text {lc }}$, allowing determination of $V_{p}^{\text {um }}$ without a precisely known $V_{p}^{\text {lc }}$ (Figs 3 and 4; also Fig. 7 in Part 1). Although $V_{p}$ um can in principle be derived from a single $S_{S} P m p$ observation, incorporating $\Phi_{\mathrm{VDSs}}$ from multiple events offers a more robust estimation of $V_{p}$ um, especially in the presence of noise (Figs 5 and S4). We note that, when measuring $\Phi_{\text {VDSS }}$, it is important to choose traces with high signal-to-noise ratio, as waveform observables are more sensitive to noise than traveltime measurements. This is especially true when considering the higher noise level of teleseismic $S$ compared to $P$ wave due to coda waves generated by preceding $P$ phases that arrive in the same time window. A practical criterion for data quality control is to choose SsPmp traces that can be well-fitted with a phase-shifted $S s$ wavelet (Fig. 9). Our field examples show that $\Phi_{\text {VDSs }}$ and $T_{\text {VDSs }}$ of events selected this way (cyan markers in Fig. S6) in general have smaller uncertainties than the events not selected (grey markers in Fig. S6).
While inferring $V_{p}{ }^{\text {um }}, H_{\mathrm{VDSS}}$ and $V_{p}{ }^{\text {av }}$ from $\Phi_{\mathrm{VDSS}}$ and $T_{\mathrm{VDSS}}$ observations, we implicitly assume the CMB to be a sharp boundary as opposed to a broad transition zone. When the CMB is a velocitygradient zone thinner than the dominant wave-length of the incident $P$ wave ( $\sim 25 \mathrm{~km}$ for typical field data), as is true for most continental areas, $\Phi_{\text {VDSs }}$ is not significantly different from the case with a sharp CMB (Part 1), thus our method of deriving $V_{p}{ }^{\text {um }}$ from $\Phi_{\text {VDSs }}$ still applies. When the CMB is a velocity-gradient zone with $S s P m p$ rays turning within the $\mathrm{CMB}(1 / p$ in the range of the gradient-zone $V_{p}$ ), the $T_{\mathrm{VDSS}}-p$ relation (moveout) is different from the case with a sharp CMB (Fig. 9 in Part 1), making our method of deriving $H_{\text {VDSS }}$ and $V_{p}$ av from $T_{\text {VDSS }}$ less accurate. However, the very good agreement between $H_{\mathrm{VDSS}}$ and $H_{H \kappa}$ (Fig. 10c) for the SW Slave Craton not only demonstrates the robustness of our method, but also verifies the underlying assumptions that we make about the study area. First, the study area is well approximated with a 1-D lithospheric model. Secondly, the CMB beneath the study area is 
sufficiently sharp that $S s P m p$ reflections and $P S$ conversions happen at essentially the same depth.

\section{CONCLUSIONS}

We have shown with synthetic and field examples that $T_{\mathrm{VDSS}}$ and $\Phi_{\text {VDSs }}$ can be used to derive average crustal $V_{p}$, crustal thickness and $V_{p}$ of the uppermost mantle. We have also demonstrated that average crustal $V_{p} / V_{s}$ ratio and composition can be estimated with PRF-VDSS joint analysis. Our field example from the SW Slave Craton shows an intermediate crustal composition, together with crustal thickness suggestive of a Mesoarchean formation age.

\section{ACKNOWLEDGEMENTS}

We thank Brad Hacker from University of California, Santa Barbara, who provided his compilation of laboratory measurements. We thank the Data Management Center of the Incorporated Research Institutions for Seismology (IRIS-DMC) for the waveforms of the Yellowknife array and Canadian Hazards Information Service for the data of the POLARIS array. We also thank Jieyuan Ning from Peking University and two anonymous reviewers for their valuable advice. Tianze Liu is supported by a Stanford Graduate Fellowship. Gabriel Ferragut was supported by an IRIS internship during his stay at Stanford.

\section{REFER E N C ES}

Aki, K. \& Richards, P.G., 2002a. Phase shifts: phase delay and phase advance, in Quantitative Seismology, 2nd edn, p. 151, ed. Ellis, J., University Science Books.

Aki, K. \& Richards, P.G., 2002b. Reflection and transmission of P-SV across a solid-solid interface, in Quantitative Seismology, 2nd edn, pp. 139-145, ed. Ellis, J., University Science Books.

Barruol, G. \& Kern, H., 1996. Seismic anisotropy and shear-wave splitting in lower-crustal and upper-mantle rocks from the Ivrea Zone-experimental and calculated data, Phys. Earth planet. Inter., 95, 175-194.

Bennett, V., Jackson, V.A., Rivers, T., Relf, C., Horan, P. \& Tubrett, M., 2005. Geology and U-Pb geochronology of the Neoarchean Snare River terrane: tracking evolving tectonic regimes and crustal growth mechanisms, Can. J. Earth Sci., 42, 895-934.

Bleeker, W., 2002. Archaean tectonics: a review, with illustrations from the Slave craton, Geol. Soc. Lond., Spec. Publ., 199, 151-181.

Bostock, M.G., 1998. Mantle stratigraphy and evolution of the Slave province, J. geophys. Res., 103, 21 183-21 200.

Bowring, S.A. \& Williams, I.S., 1999. Priscoan (4.00-4.03 Ga) orthogneisses from northwestern Canada, Contrib. to Mineral. Petrol., 134, $3-16$.

Christensen, N.I., 1996. Poisson's ratio and crustal seismology, J. geophys. Res., 101, 3139-3156.

Christensen, N.I. \& Mooney, W.D., 1995. Seismic velocity structure and composition of the continental crust: a global view, J. geophys. Res. Solid Earth, 100, 9761-9788.

Fernández-Viejo, G. \& Clowes, R.M., 2003. Lithospheric structure beneath the Archaean Slave Province and Proterozoic Wopmay orogen, northwestern Canada, from a LITHOPROBE refraction/wide-angle reflection survey, Geophys. J. Int., 153, 1-19.

Fernández-Viejo, G., Clowes, R.M. \& Welford, J.K., 2005. Constraints on the composition of the crust and uppermost mantle in northwestern Canada: $V p / V s$ variations along Lithoprobe's SNorCLE transect, Can. J. Earth Sci., 42, 1205-1222.

Hacker, B.R., Kelemen, P.B. \& Behn, M.D., 2015. Continental Lower Crust, Annu. Rev. Earth planet. Sci., 43, 167-205.

Hammer, P.T.C., Clowes, R.M., Cook, F.A., van der Velden, A.J. \& Vasudevan, K., 2010. The Lithoprobe trans-continental lithospheric cross sections: imaging the internal structure of the North American continent, Can. J. Earth Sci., 47, 821-857.

Herrmann, R.B., 2013. Computer programs in seismology: an evolving tool for instruction and research, Seismol. Res. Lett., 84, 1081-1088.

Kang, D., Yu, C., Ning, J. \& Chen, W., 2016. Simultaneous determination of crustal thickness and $P$ wavespeed by Virtual Deep Seismic Sounding (VDSS), Seismol. Res. Lett., 87, 1104-1111.

Karplus, M.S. et al., 2019. Receiver-function imaging of the lithosphere at the Kunlun-Qaidam boundary, Northeast Tibet, Tectonophysics, 759, $30-43$.

Liu, T., Klemperer, S.L., Yu, C. \& Ning, J., 2018. Post-critical SsPmp and its applications to Virtual Deep Seismic Sounding (VDSS)-1: sensitivity to lithospheric 1-D and 2-D structure, Geophys. J. Int., 215, 880-894.

Luo, S., Zhu, L., Huang, R., Luo, Y., Jiang, X. \& Hua, Y., 2018. Determination of crustal thickness and velocities by using receiver functions and PmP travel times, Geophys. J. Int., 216, 1304-1312.

Parker, E.H., Hawman, R.B., Fischer, K.M. \& Wagner, L.S., 2016. Estimating crustal thickness using SsPmp in regions covered by low-velocity sediments: imaging the moho beneath the Southeastern Suture of the Appalachian Margin Experiment (SESAME) array, SE Atlantic Coastal Plain, Geophys. Res. Lett., 43, 9627-9635.

Porritt, R.W. \& Miller, M.S., 2018. Updates to FuncLab, a Matlab based GUI for handling receiver functions, Comput. Geosci., 111, 260-271.

Randall, G.E., 1989. Efficient calculation of differential seismograms for lithospheric receiver functions, Geophys. J. Int., 99, 469-481.

Snyder, D. \& Bruneton, M., 2007. Seismic anisotropy of the Slave craton, NW Canada, from joint interpretation of SKS and Rayleigh waves, Geophys. J. Int., 169, 170-188.

Thompson, D.A., Rawlinson, N. \& Tkalčić, H., 2019. Testing the limits of virtual deep seismic sounding via new crustal thickness estimates of the Australian continent, 218(2), Geophys. J. Int., 787-800.

Tian, X. et al., 2015. Weakly coupled lithospheric extension in southern Tibet, Earth planet. Sci. Lett., 430, 171-177.

Tseng, T.L., Chen, W.P. \& Nowack, R.L., 2009. Northward thinning of Tibetan crust revealed by virtual seismic profiles, Geophys. Res. Lett., 36, $1-5$.

van der Velden, A.J. \& Cook, F.A., 2002. Products of 2.65-2.58 Ga orogenesis in the Slave Province correlated with Slave-Northern Cordillera Lithospheric Evolution (SNORCLE) seismic reflection patterns, Can. J. Earth Sci., 39, 1189-1200.

Yu, C., Chen, W.P. \& van der Hilst, R.D., 2016. Constraints on residual topography and crustal properties in the western United States from virtual deep seismic sounding, J. geophys. Res.: Solid Earth, 121, 5917-5930.

Yu, C.Q., Chen, W.P., Ning, J.Y., Tao, K., Tseng, T.L., Fang, X.D., John Chen, Y. \& van der Hilst, R.D., 2012. Thick crust beneath the Ordos plateau: implications for instability of the North China craton, Earth planet. Sci. Lett., 357-358, 366-375.

Yu, C.Q., Chen, W.P. \& Van Der Hilst, R.D., 2013. Removing source-side scattering for virtual deep seismic sounding (VDSS), Geophys. J. Int., 195, 1932-1941.

Yuan, H., 2015. Secular change in Archaean crust formation recorded in Western Australia, Nat. Geosci., 8, 808-813.

Zhu, L. \& Kanamori, H., 2000. Moho depth variation in southern California from teleseismic receiver functions, J. geophys. Res., 105, 2969-2980.

\section{SUPPORTING INFORMATION}

Supplementary data are available at $G J I$ online.

Text S1. Estimating uncertainties of $\Phi_{\mathrm{VDSS}}$ and $T_{\mathrm{VDSS}}$.

Table S1. Information of Grade A and B events recorded at YKW3.

Figure S1. Constraining Moho depth and $V_{p}$ um using synthetic data with 10 per cent white noise. 
Figure S2. Distribution of $V_{p}$ um derived from random simulations of noisy synthetic SsPmp waveforms.

Figure S3. Uncertainty estimation for $\Phi_{\text {VDSs }}$.

Figure S4. Constraining Moho depth, $V_{p}^{\text {av }}$ and $V_{p}^{\text {um }}$ using synthetic data with 10 per cent white noise.

Figure S5. PRF observations for EDZN.
Figure S6. Effects of data selection on estimated Moho depth, $V_{p}^{\text {av }}$ and $V_{p}$ um , for real data.

Please note: Oxford University Press is not responsible for the content or functionality of any supporting materials supplied by the authors. Any queries (other than missing material) should be directed to the corresponding author for the paper. 